\title{
Two-Field Transverse Relaxation-Optimized Spectroscopy for the Study of Large Biomolecules - an in silico investigation
}

\author{
Nicolas Bolik-Coulon, Philippe Pelupessy, Guillaume Bouvignies*, Fabien Ferrage*
}

\author{
Addresses \\ Laboratoire des Biomolécules, LBM, Département de chimie, École normale supérieure, PSL \\ University, Sorbonne Université, CNRS, 75005 Paris, France \\ *Corresponding authors : Guillaume.Bouvignies@ens.psl.eu and Fabien.Ferrage@ens.psl.eu
}

\begin{abstract}
Biomolecular NMR spectroscopy has greatly benefited from the development of TROSY-type pulse sequences, in pair with specific labeling. The selection of spin operators with favorable relaxation properties has led to an increase in the resolution and sensitivity of spectra of large biomolecules. However, nuclei with a large chemical shift anisotropy (CSA) contribution to relaxation can still suffer from large linewidths at conventional magnetic fields (higher than 9 T). Here, we introduce the concept of two-field TROSY (2F-TROSY) where the chemical shifts of nuclei with large CSA is labeled at low fields (ca. $2 \mathrm{~T})$ dramatically reducing the contribution of CSA to relaxation. Signal detection is performed at high field $(>9 \mathrm{~T})$ on a nucleus with efficient TROSY interference to yield high-resolution and sensitivity. We use comprehensive numerical simulations to demonstrate the power of this approach on aromatic ${ }^{13} \mathrm{C}-{ }^{19} \mathrm{~F}$ spin pairs for which a TROSY pulse sequence has recently been published. We predict that the $2 \mathrm{~F}$ TROSY experiment shall yield good quality spectra for large proteins (global tumbling correlation times as high as $100 \mathrm{~ns}$ ) with one order of magnitude higher sensitivity than the single-field experiment.
\end{abstract}

\section{keywords}

Two-Field NMR, Protein NMR, TROSY, Fluorine NMR, aromatic side chains 


\section{INTRODUCTION}

Nuclear Magnetic Resonance (NMR) is a powerful tool to investigate the structure, dynamics and function of complex biomolecular systems at atomic resolution. The use of advanced isotopic labeling methods has facilitated the study of large biomolecules and biomolecular complexes by focusing on a limited number of well-defined spin systems, such as methyl groups in aliphatic side-chains. ${ }^{1,2}$ Selective isotope labeling is optimally suited to the investigation of large systems when combined with transverse relaxation-optimized spectroscopy (TROSY) methods. First introduced for backbone ${ }^{15} \mathrm{~N}-{ }^{1} \mathrm{H}$ pairs in deuterated proteins, ${ }^{3}$ and a variety of similar two-spin one half systems in protein sidechains and nucleic acids, ${ }^{4-6}$ the concept of TROSY was later successfully extended to methyl groups as encountered in protein side-chains ${ }^{7,8}$ or methylated nucleic acids. ${ }^{9}$

The NH- and methyl-TROSY both rely on interference between relaxation mechanisms. The methyl-TROSY experiment is based on the cancellation between intra-methyl dipole-dipole (DD) interactions while the NH-TROSY exploits interference between DD and Chemical Shift Anisotropy (CSA) interactions. ${ }^{7,10-12}$ The amplitude of the dipolar interaction is fieldindependent so that the methyl-TROSY effect is ubiquitous at conventional magnetic fields $\left(\mathrm{B}_{0}\right.$ $>9 \mathrm{~T}$ ) and even at lower magnetic fields, down to a fraction of a Tesla. ${ }^{13}$ The CSA interaction scales linearly with the magnetic field and, consequently, TROSY effects based on DD/CSA interference mechanisms are optimal at magnetic fields where the CSA interactions has the same magnitude as the DD interactions. ${ }^{3}$ However, recent theoretical work showed that the optimal field in terms of signal-to-noise ratio for NH-TROSY is $1.5 \mathrm{GHz}$ proton Larmor frequency, whereas optimal DD/CSA interference is around $950 \mathrm{MHz}$, as higher fields lead to better intrinsic sensitivity. ${ }^{14}$ In two-spin systems, an essential component to obtain increased resolution is the selection of the appropriate operator (the TROSY single-transition operator) at the optimal field.

The set of two-spin TROSY pulse sequences used for the study of biomolecules has mainly been applied on pairs of the type $\mathrm{X}-{ }^{1} \mathrm{H}\left(\mathrm{X}=\right.$ backbone- $-{ }^{15} \mathrm{~N}$ in proteins or aromatic- ${ }^{15} \mathrm{~N}$ and ${ }^{13} \mathrm{C}$ in proteins and nucleic acids)..$^{3-6}$ In these spin systems, the CSA of the protons is either small or comparable to the amplitude of the DD interaction at magnetic fields currently accessible (between 9 and $28 \mathrm{~T}$ ) and leads to field-dependence of the proton transverse relaxation rate usually less pronounced than for the relaxation of backbone- $-{ }^{15} \mathrm{~N}$ and aromatic- ${ }^{13} \mathrm{C}$ nuclei. ${ }^{14}$ Thus, the optimal field for the associated TROSY experiment depends mostly on the relaxation properties of the heteronucleus. This is not the case in the recently developed two-dimensional ${ }^{13} \mathrm{C}-{ }^{19} \mathrm{~F}-\mathrm{TROSY}$ experiment for the study of specifically ${ }^{19} \mathrm{~F}$-labeled protein aromatic side- 
chains and nucleic acid bases. ${ }^{15,16}$ The potential of this approach cannot be overstated, as the study of aromatic side chains by NMR is one of the biggest challenges in large biomolecules. The interference between the ${ }^{13} \mathrm{C}-{ }^{19} \mathrm{~F}$ DD and the ${ }^{13} \mathrm{C}-\mathrm{CSA}$ relaxation mechanisms is strong, leading to favorable relaxation properties of the TROSY component of the ${ }^{13} \mathrm{C}$ polarization. On the other hand, because of the large ${ }^{19} \mathrm{~F}-\mathrm{CSA}$, the interference between the ${ }^{13} \mathrm{C}-{ }^{19} \mathrm{~F}$ DD and the ${ }^{19} \mathrm{~F}-\mathrm{CSA}$ relaxation mechanisms is far from optimal at conventional magnetic fields for biomolecular NMR (higher than 9.4 T). The unfavorable ${ }^{19} \mathrm{~F}$ relaxation properties originating from its large CSA lead to two drastically different optimal fields for the relaxation of ${ }^{19} \mathrm{~F}$ and ${ }^{13} \mathrm{C}$ coherences. ${ }^{15}$ Is it nevertheless possible to define a magnetic field that would be a good compromise for aromatic ${ }^{13} \mathrm{C}-{ }^{19} \mathrm{~F}$-TROSY experiments in large proteins (with a correlation time for overall rotational diffusion $\tau_{\mathrm{c}}>25 \mathrm{~ns}$ ) ? Numerical simulations (see below) show that there will likely be no good single magnetic field compromise for the investigation of large systems by ${ }^{13} \mathrm{C}-{ }^{19} \mathrm{~F}-\mathrm{TROSY}$. Then, would it be possible to exploit the optimal relaxation properties of both ${ }^{13} \mathrm{C}$ and ${ }^{19} \mathrm{~F}$ within the same experiment?

Here, we introduce the concept of two-field transverse relaxation-optimized spectroscopy (2FTROSY) where we exploit the different optimums for the transverse relaxation in multiple-spin systems by visiting two vastly different magnetic fields within a single experiment. The $2 \mathrm{~F}$ TROSY experiment can be implemented on a two-field NMR spectrometer, where two magnetic centers equipped with probes are coupled by a sample shuttle. A prototype of twofield NMR spectrometer ${ }^{17}$ has already been used to overcome chemical exchange broadening, ${ }^{18}$ obtain broad-band correlations throughout aliphatic and aromatic ${ }^{13} \mathrm{C}$ resonances ${ }^{19}$ and measure accurate relaxation rates at low field. ${ }^{20,21}$

We use numerical simulations to illustrate the concept of $2 \mathrm{~F}-\mathrm{TROSY}$ on aromatic ${ }^{13} \mathrm{C}-{ }^{19} \mathrm{~F}$ spin systems. We calculate the expected spectra of aromatic fluorinated protein side-chains in a twofield experiment where the ${ }^{19} \mathrm{~F}$ coherence evolves at low field, while the ${ }^{13} \mathrm{C}$-TROSY component is detected at high field, and compare them with the reported single-field ${ }^{13} \mathrm{C}-{ }^{19} \mathrm{~F}$ TROSY experiment. ${ }^{15}$ We predict that ${ }^{13} \mathrm{C}-{ }^{19} \mathrm{~F}$ 2F-TROSY will offer better sensitivity and resolution in medium and large-size proteins $\left(\tau_{\mathrm{c}}>25 \mathrm{~ns}\right)$. We expect that 2F-TROSY will enhance NMR of large biomolecules by making a diversity of new spin systems accessible, starting with ${ }^{13} \mathrm{C}-{ }^{19} \mathrm{~F}$ pairs in aromatic side-chains.

\section{THEORY AND CALCULATIONS}

Relaxation theory. We considered pairs of directly bound ${ }^{13} \mathrm{C}$ and ${ }^{19} \mathrm{~F}$ nuclei, with the physical properties of a specifically labelled side chain of 3-Fluorotyrosine (3F-Tyr) as previously 
used. ${ }^{15}$ Analytical calculations of the relaxation rates (reported in the Supplementary Materials) for an isolated ${ }^{13} \mathrm{C}-{ }^{19} \mathrm{~F}$ spin pair were performed using the program RedKite ${ }^{22}$ with a bond length of 133.8 picometers. ${ }^{15}$ The ${ }^{13} \mathrm{C}$ - and ${ }^{19} \mathrm{~F}$-CSA tensors from Ref. ${ }^{15}$ were used and are given in Table S1. We used the model-free approach ${ }^{23}$ for the spectral density function, which includes the effect of internal motions and global tumbling:

$$
J_{\vec{\imath}, \vec{j}}(\omega)=\frac{2}{5} P_{2}\left(\cos \left(\theta_{\vec{l}, \vec{j}}\right)\right)\left[S^{2} \frac{\tau_{c}}{1+\left(\omega \tau_{c}\right)^{2}}+\left(1-S^{2}\right) \frac{\tau_{l}^{\prime}}{1+\left(\omega \tau_{l}^{\prime}\right)^{2}}\right],
$$

where $\boldsymbol{i}$ and $\boldsymbol{j}$ are vectors pointing along the principal axes of interactions $i$ and $j$ in the molecular frame, $\mathrm{P}_{2}(\mathrm{x})$ is the second order Legendre polynomial $P_{2}(x)=\left(3 x^{2}-1\right) / 2, \tau_{l}^{\prime-1}=\tau_{c}^{-1}+$ $\tau_{l}^{-1}$ with $\tau_{\mathrm{c}}$ the isotropic global tumbling correlation time and $\tau_{1}$ the correlation time associated with local motions of order parameter $\mathrm{S}^{2}$. We set the parameters of local dynamics to those of a rigid side chain, with $\mathrm{S}^{2}=0.8$ and $\tau_{1}=100$ ps. Evolutions of the relaxation rates with the magnetic field are shown in Fig. S1.

Simulation of two-dimensional spectra. A detailed description of the simulations of the spectra can be found in the Supplementary Materials. To accurately account for polarization losses, lineshapes, and pathways, the evolution of the magnetization throughout the full pulse sequence was simulated by integrating the complete master equation ${ }^{24}$ and generate the free induction decays (FID) using Python and the numpy ${ }^{25}$ and scipy $^{26}$ packages. FIDs were processed with standard methods using the nmrglue package ${ }^{27}$ (see Supplementary Materials). All recycling delays were optimized to yield the maximum signal per unit of experimental time.

Simulating a two-field NMR spectrometer. The simulation of the evolution of the density operator during the shuttle transfers was performed as previously described. ${ }^{22}$ Briefly, we used the existing prototype of a two-field NMR spectrometer operating at $14.1 \mathrm{~T}$ and $0.33 \mathrm{~T}$ to model the design of other two-field NMR systems. The field profiles were assumed to be identical to the one of our current system apart from a scaling factor $\alpha\left(B_{0}\right)$ where $\mathrm{B}_{0}$ is the high-field value and $\alpha$ is a dimensionless function of the magnetic field $B: \alpha(B)=B / 14.1 T$ (Fig. S2). In this design, the sample is shuttled at constant speed until it reaches the desired low field. The computation of the density operator is performed every $1 \mathrm{~ms}$ during the sample shuttle transfers. Scaling in noise-free spectra. To take into account the dependence of the signal-to-noise ratio (SNR) with the experimental time when comparing the different experiments, FIDs were scaled by $\sqrt{t_{P S} / t_{r e f}}$, with tps the experimental time to record one scan without evolution in the indirect dimension and $t_{\text {ref }}$ the duration of the experiment used as a reference. We choose as reference experiment the single-field TROSY pulse sequence ${ }^{15}$ (Fig. S3) used on a protein of global 
tumbling correlation time $\tau_{\mathrm{c}}=25 \mathrm{~ns}$ labelled with 3F-Tyr. The proportionality of the polarization with $\mathrm{B}_{0}$ was considered by including a field-dependent thermal correction ${ }^{28}$ in the Liouvillian (see Supplementary Materials), while the effect of the magnetic field on the SNR was included by multiplying the FID by $\sqrt{\alpha\left(B_{0}\right)}$.

Scaling spectra with noise. When mentioned, Gaussian white noise was added to the simulated FID. In these cases, FID and random noise were scaled with $n \times \alpha\left(B_{0}\right)$ and $\sqrt{n \times \alpha\left(B_{0}\right)}$ respectively, where $n$ is the number of scans needed for the experimental time to be identical to the reference single-field TROSY experiment with 80 scans and $t_{1, \max }$ given by $1.25 \mathrm{xT}_{2}\left({ }^{19} \mathrm{~F}\right.$ TROSY) with $\mathrm{T}_{2}\left({ }^{19} \mathrm{~F}-\mathrm{TROSY}\right)$ the relaxation time for the ${ }^{19} \mathrm{~F}$-TROSY component (see Section 1.3 of the Supplementary Materials).

\section{RESULTS AND DISCUSSION}

Relaxation properties of aromatic ${ }^{13} \mathbf{C}-{ }^{19} \mathbf{F}$ groups. The large CSA (Table S1) is the dominant source of ${ }^{19} \mathrm{~F}$ nuclear spin relaxation at high magnetic fields (i.e. higher than $10 \mathrm{~T}$ ). The CSA contribution to transverse relaxation scales with the square of the magnetic field and becomes a minor contribution at fields lower than a few Tesla (7\% at $1 \mathrm{~T}$ vs $95 \%$ at $14.1 \mathrm{~T}$ for the $3 \mathrm{~F}$ Tyr and $\tau_{\mathrm{c}}=25 \mathrm{~ns}$ ) where relaxation is mainly caused by the dipole-dipole (DD) interaction. Fast ${ }^{19} \mathrm{~F}$ transverse relaxation, both for an in-phase coherence and TROSY single-transition, precludes the observation of intense and well-resolved peaks at magnetic fields that are commonly used for biomolecules (Fig. 1.a). The ${ }^{13} \mathrm{C}$-TROSY component displays very favorable relaxation properties at high-field: at $14.1 \mathrm{~T}$, the TROSY effect is strong with a relaxation rate for the TROSY component about 6 times lower than the in-phase transverse relaxation rate (Fig. 1.b). The most favorable transverse relaxation properties are found at low magnetic fields $\left(\mathrm{B}_{0} \sim 1 \mathrm{~T}\right)$ for ${ }^{19} \mathrm{~F}$ and at high magnetic field $\left(\mathrm{B}_{0} \sim 15 \mathrm{~T}\right)$ for ${ }^{13} \mathrm{C}$ for proteins with correlation times in the range 1 to $200 \mathrm{~ns}$. These two drastically different optimal magnetic fields suggest that any single field ${ }^{14}$ for the ${ }^{13} \mathrm{C}-{ }^{19} \mathrm{~F}$ TROSY experiment ${ }^{15}$ (hereafter referred to as 1F-TROSY) will likely be a bad compromise between irreconcilable constraints (Fig. S4). By contrast, two-field NMR offers in principle the possibility to reach two independently optimized magnetic fields within the course of a single experiment. This approach should be particularly well suited to record the spectra of aromatic ${ }^{13} \mathrm{C}-{ }^{19} \mathrm{~F}$ groups. 

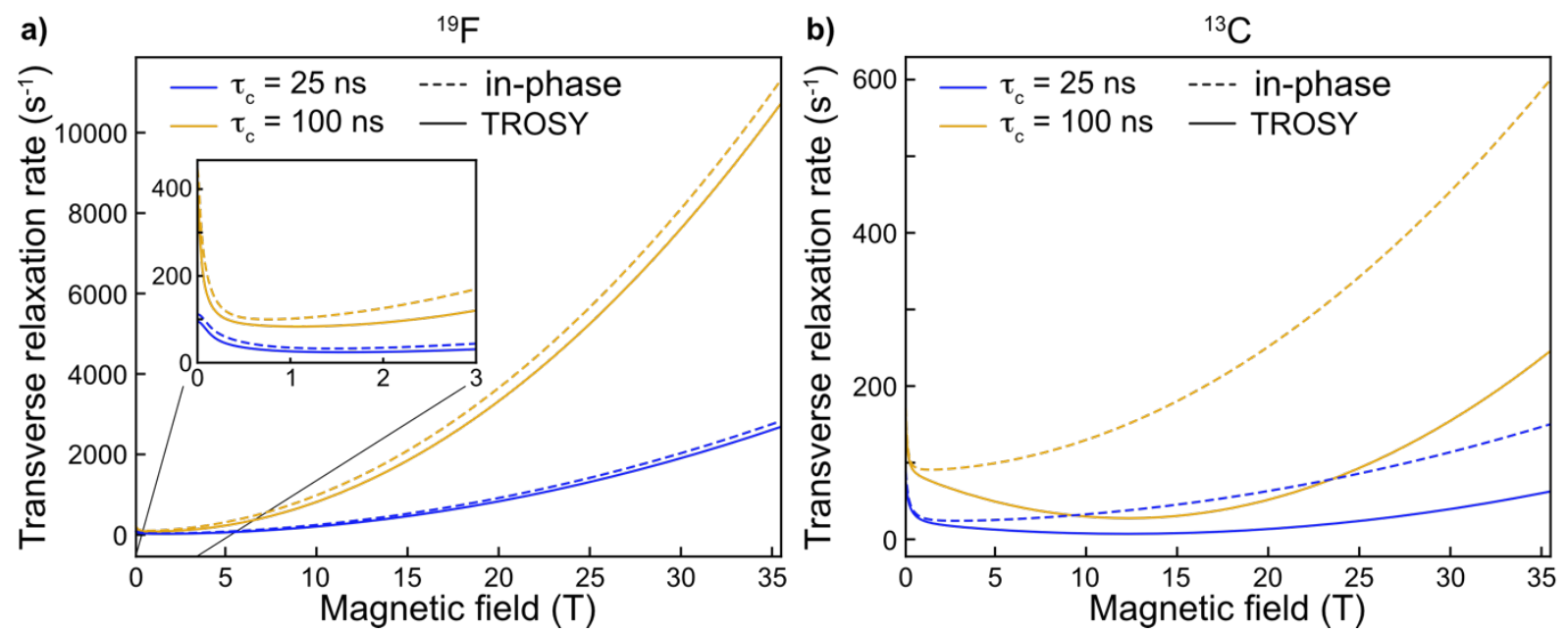

Figure 1: Transverse relaxation rates of an isolated ${ }^{13} \mathrm{C}-{ }^{19} \mathrm{~F}$ aromatic spin pair. Fluorine-19 (a) and carbon-13 (b) relaxation rates of the in-phase coherences (dash) and TROSY component (plain) for $\tau_{\mathrm{c}}=25 \mathrm{~ns}$ (blue) and $\tau_{\mathrm{c}}=100 \mathrm{~ns}$ (orange). Calculations were performed using the parameters for the CSA tensors of 3F-Tyr.

Pulse sequence for two-field TROSY. The two-field two-dimensional heteronuclear correlation pulse sequence (referred to as 2F-TROSY) proposed here is presented in Fig. 2. Nuclear spin systems are controlled using radiofrequency pulses and pulsed field gradients at both high- and low-field magnetic centers. Additional delays corresponding to sample shuttle transfers from one magnetic center to the other $\left(\tau_{\mathrm{Sh}}\right)$ and waiting delays needed by the shuttle apparatus before $\left(\tau_{\mathrm{HF}, 1}\right.$ and $\left.\tau_{\mathrm{LF}, 2}\right)$ and after shuttling $\left(\tau_{\mathrm{LF}, 1}\right.$ and $\left.\tau_{\mathrm{HF}, 2}\right)$ were included when simulating the pulse sequence. ${ }^{17}$

The sample is polarized at high field before shuttling to low field. At point a of the pulsesequence, $F_{z}$ is the only operator with a non-zero expectation value. The ${ }^{19} \mathrm{~F}$ chemical shift is labeled at low field in a semi-constant time fashion ${ }^{29}$ with an effective evolution under the ${ }^{13} \mathrm{C}$ ${ }^{19} \mathrm{~F}$ scalar-coupling for a duration $\mathrm{T}=\left(2\left|\mathrm{~J}_{\mathrm{CF}}\right|\right)^{-1}$. Depending on the phase $\varphi_{1}$, either the cosine or sine $\mathrm{t}_{1}$-evolving components are stored as a two-spin order $2 F_{z} C_{z}$ (point $\mathbf{b}$ ) and preserved during the transfer from low to high magnetic field (from $\mathbf{b}$ to $\mathbf{c}$ ). The spin-state-selective excitation (S3E) block ${ }^{30}$ allows for efficient selective excitation of the ${ }^{13} \mathrm{C}$-TROSY component at high field (point d). The use of two inversion pulses on ${ }^{19} \mathrm{~F}$ at high field preserves the longitudinal magnetization recovered between points $\mathbf{b}$ and $\mathbf{c}$. This scheme allows to reduce the subsequent recycling delay and make the experiment more time efficient. When ${ }^{19} \mathrm{~F}$ broadband excitation is needed, composite $\pi$-pulses can be used, keeping in mind that transverse relaxation may not be negligible during ${ }^{19} \mathrm{~F}$ pulses (Fig. 1a).

Throughout the phase cycle, the nature of the evolving operators during the $\mathrm{S}^{3} \mathrm{E}^{30}$ element is the same, ensuring identical relaxation properties of the different pathways during the $\mathrm{S}^{3} \mathrm{E}^{30}$ block and the effective cancellation of undesired components of the density operator. This is 
not the case for the 1F-TROSY pulse sequence ${ }^{15}$ for which the single transition-to-single transition polarization transfer block $^{31}$ (ST2-PT) creates alternatively a single-quantum antiphase ${ }^{13} \mathrm{C}$ coherence and a multiple-quantum transition coherence which relax with drastically different rates at high fields because of the strong ${ }^{19} \mathrm{~F}-\mathrm{CSA}$ (see Supplementary Materials for a derivation of the pulse sequence and Fig. S1). This leads to poor selection of the TROSY line in the original ${ }^{13} \mathrm{C}-{ }^{19} \mathrm{~F}$ TROSY experiment (Fig. S5). Similarly, an alternative 2F-TROSY pulse sequence could select the ${ }^{19} \mathrm{~F}-\mathrm{TROSY}$ component with an ST2-PT ${ }^{31}$ block at low field (Fig. S6). Efficient cross-relaxation between the two ${ }^{13} \mathrm{C}$ single-transition longitudinal polarization operators during the shuttle transfer and stabilization delays would lead to poor TROSY selection and thus the use of ST2-PT for 2F-TROSY is not recommended (Fig. S7 and S8).

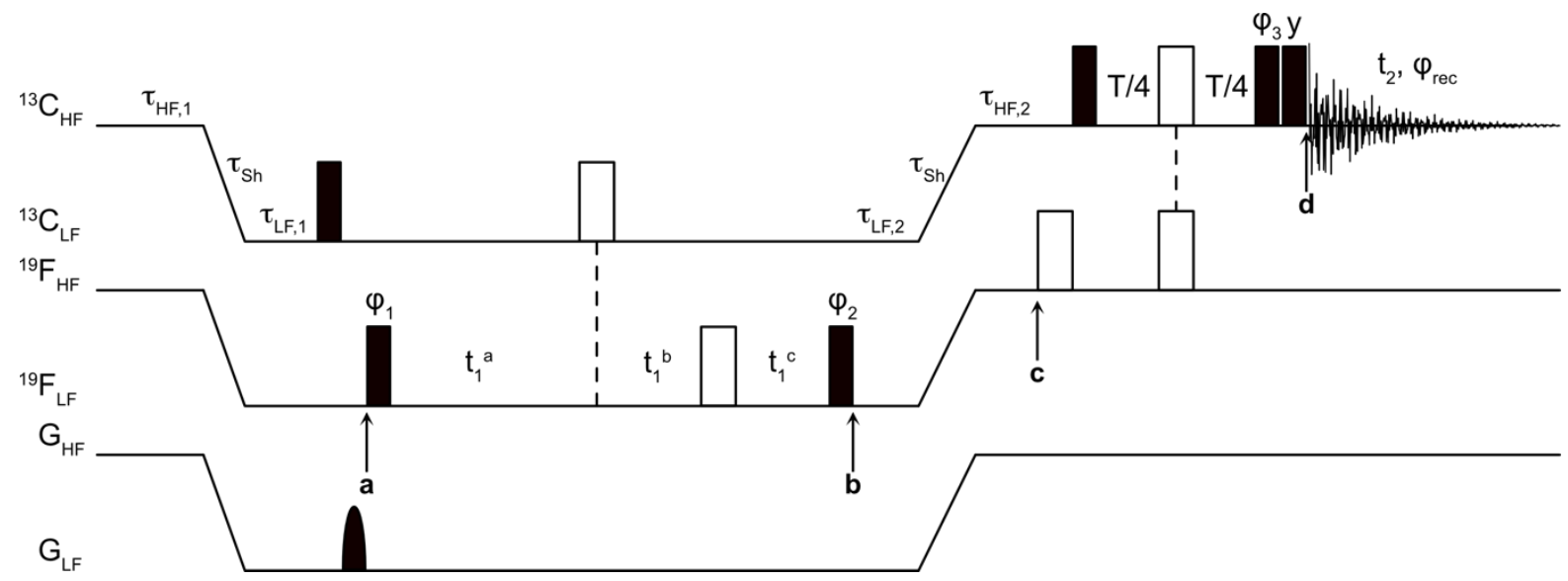

Figure 2: Two-field transverse relaxation-optimized spectroscopy pulse sequence for the study of aromatic ${ }^{13} \mathrm{C}-{ }^{19} \mathrm{~F}$ groups $(2 \mathrm{~F}-$ TROSY). Black narrow (respectively wide white) rectangles represent $90^{\circ}$ (respectively $180^{\circ}$ ) pulses. Phases are aligned along the $\mathrm{x}$-axis of the rotating frame unless otherwise stated. Phase cycles are as follows: $\varphi_{1}=(x,-x, x,-x, x,-x, x,-x), \varphi_{2}=$ $(y, y, y, y,-y,-y,-y,-y), \varphi_{3}=\left(\frac{\pi}{4}, \frac{\pi}{4}, \frac{5 \pi}{4}, \frac{5 \pi}{4}, \frac{\pi}{4}, \frac{\pi}{4}, \frac{5 \pi}{4}, \frac{5 \pi}{4}\right)$ and $\varphi_{r e c}=(x,-x,-x, x,-x, x, x,-x)$. The sign discrimination of the frequency in the indirect dimension is achieved using the States method by changing the phase $\varphi_{1}$ to $(y,-y, y,-y, y,-y, y,-y)$. For the semi-constant time indirect evolution period, $t_{1}^{a}=\frac{T}{2}+\frac{n_{1}}{N-1} \frac{t_{1, \max }}{2}, t_{1}^{b}=\frac{n_{1}}{2(N-1)}\left(t_{1, \max }-\right.$ $T$ ) and $t_{1}^{c}=\frac{T}{2} \frac{N-n_{1}-1}{N-1}$, with $T=\frac{1}{2\left|J_{C F}\right|}, \mathrm{N}$ and $\mathrm{t}_{1, \max }$ the number of points and maximum evolution delay in the indirect dimension respectively, $\mathrm{n}_{1}$ the increment number. The pulse sequence can be modified to include proton decoupling during the $t_{1}$ and $t_{2}$ evolution periods. The horizontal line breaks represent the shuttling transfers from one field to the other. $\tau_{\mathrm{Sh}}$ is the shuttling delay to and from the desired position. $\tau_{\mathrm{HF}, 1}$ and $\tau_{\mathrm{HF}, 2}$ are waiting delays at high field, respectively before and after shuttling. $\tau_{\mathrm{LF}, 1}$ and $\tau_{\mathrm{LF}, 2}$ are waiting delays at low field, respectively after and before shuttling. In our simulations, we set $\tau_{\mathrm{Sh}}=$ $100 \mathrm{~ms}, \tau_{\mathrm{HF}, 1}=25 \mathrm{~ms}, \tau_{\mathrm{LF}, 1}=40 \mathrm{~ms}, \tau_{\mathrm{LF}, 2}=5 \mathrm{~ms}$ and $\tau_{\mathrm{HF}, 2}=350 \mathrm{~ms}$.

What are the optimal magnetic fields to record the TROSY experiments? In order to account for all sources of polarization loss in the course of an experiment, accurate determination of the optimal magnetic fields requires the full simulation of the pulse-sequences. We compared the expected peak height at each magnetic field (or pairs of magnetic fields in the case of the 2F-TROSY sequence) assuming ideal sampling in both dimensions so that the linewidth in both dimensions is directly linked to their associated transverse relaxation rate and does not depend on the number of points in the time dimensions and apodization function (see 
Supplementary Materials for more details). In these calculations, peak heights are scaled by $\sqrt{\alpha\left(B_{0}\right) t_{P S} / t_{r e f}}$ (see method section), a factor proportional to the expected evolution of the SNR with the magnetic field and experimental time. Note that the effect of signal accumulation arising from indirect dimension acquisition is not taken into account and, therefore, peak heights do not fully reproduce expected SNR variations.
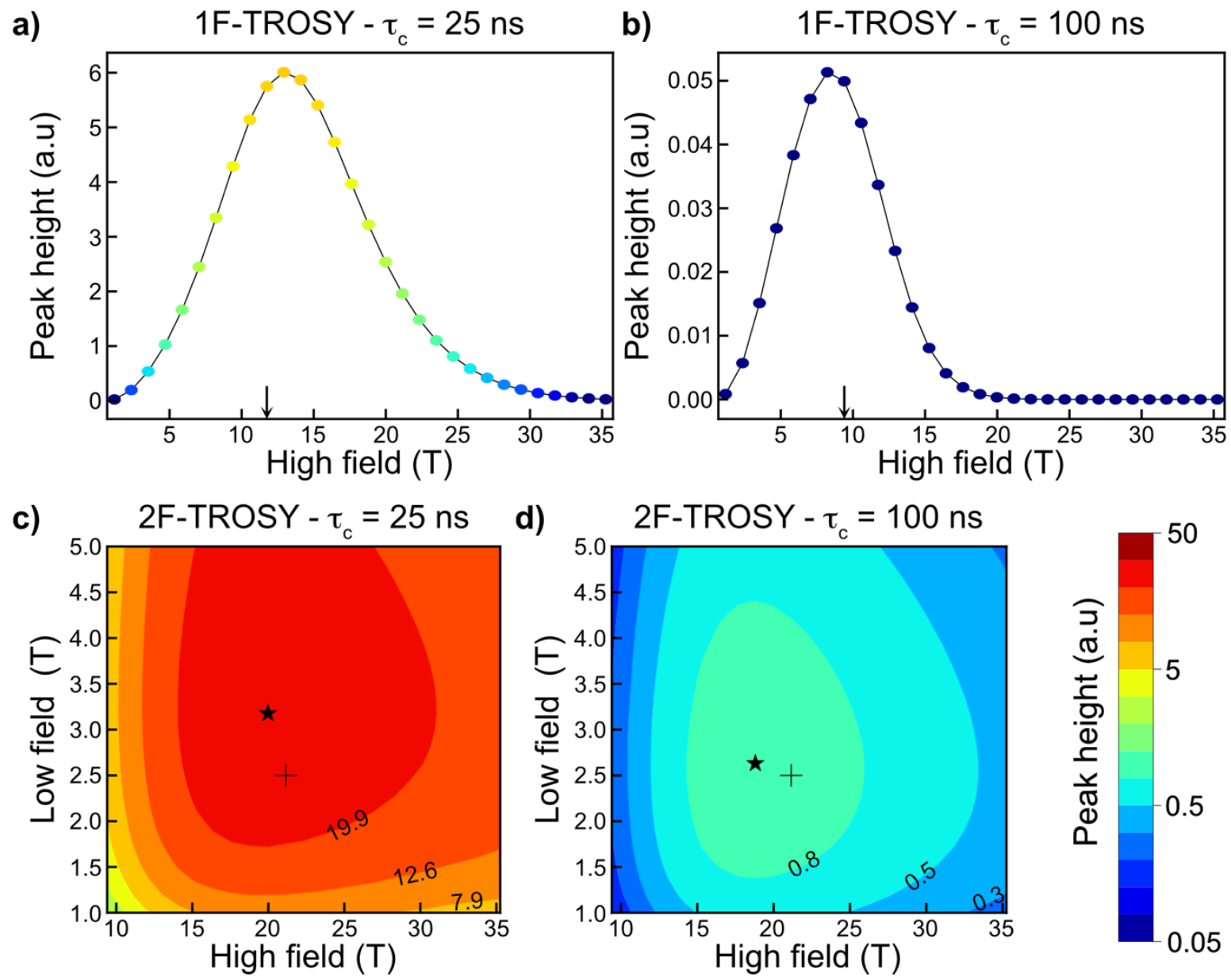

Figure 3: Magnetic field optimization of the 1F-TROSY and 2F-TROSY experiments. Expected peak heights for the 1FTROSY $(\mathbf{a}, \mathbf{b})$ and 2F-TROSY $(\mathbf{c}, \mathbf{d})$ experiments, for 3F-Tyr and a global tumbling correlation time $\tau_{\mathrm{c}}=25 \mathrm{~ns}(\mathbf{a}, \mathbf{c})$ and $\tau_{\mathrm{c}}=$ $100 \mathrm{~ns}(\mathbf{b}, \mathbf{d})$. The color scale is identical for all pannels. The conventional magnetic fields closest to the optimal fields are indicated with an arrow $(\mathbf{a}, \mathbf{d})$ or a cross $(\mathbf{c}, \mathbf{d})$. The highest peak-height position is indicated with a star in pannels $\mathbf{c}$ and $\mathbf{d}$. In pannels $\mathbf{a}$ and $\mathbf{b}$, points are connected by a solid line for visual clarity.

The optimal field for the 1F-TROSY is lower than the optimal field for ${ }^{13} \mathrm{C}$-TROSY (Fig. 3a,b) because of the losses from fast relaxation of ${ }^{19} \mathrm{~F}$-coherences during the ST2-PT ${ }^{31}$ block, as well as the broadening in the fluorine dimension caused by fast relaxation of the TROSY singletransition ${ }^{19} \mathrm{~F}$-coherence (Fig. 1a). The optimal conventional fields are predicted to be $11.75 \mathrm{~T}$ for medium sized proteins $\left(\tau_{\mathrm{c}}=25 \mathrm{~ns}\right)$, and $9.4 \mathrm{~T}$ for larger systems $\left(\tau_{\mathrm{c}}=100 \mathrm{~ns}\right)$. The optimal magnetic field for detection of the ${ }^{13} \mathrm{C}$-TROSY operator is higher in the 2F-TROSY experiment (Fig. 3c,d) since no fluorine coherence is generated at high field. For this experiment, we recommend a high field of $21.15 \mathrm{~T}$ and a low field of $2.5 \mathrm{~T}$ for the 3F-Tyr. 
In the 2F-TROSY experiment, the optimal high-field strongly correlates with the orientation of the ${ }^{13} \mathrm{C}-\mathrm{CSA}$ tensor with respect to the C-F bond (Fig. S9 and S10). A perfect alignment of the ${ }^{13} \mathrm{C}$-CSA with the C-F bond leads to an optimal interference between the CSA and DD interaction. In this case, the ${ }^{13} \mathrm{C}$-TROSY effect is strong and signal detection at higher magnetic field (leading to higher sensitivity) does not counter-balance the less efficient TROSY effect. The optimal low field for ${ }^{19} \mathrm{~F}$ chemical shift evolution inversely correlates with the value of the orthogonal component of the ${ }^{19} \mathrm{~F}-\mathrm{CSA}$ tensor (i.e. the larger component of the tensor). This leads to a higher reduction of the ${ }^{19} \mathrm{~F}-\mathrm{CSA}$ contribution to the relaxation of the evolving fluorine in-phase coherence for compounds having the larger orthogonal component of the ${ }^{19} \mathrm{~F}-\mathrm{CSA}$ tensor.

a)

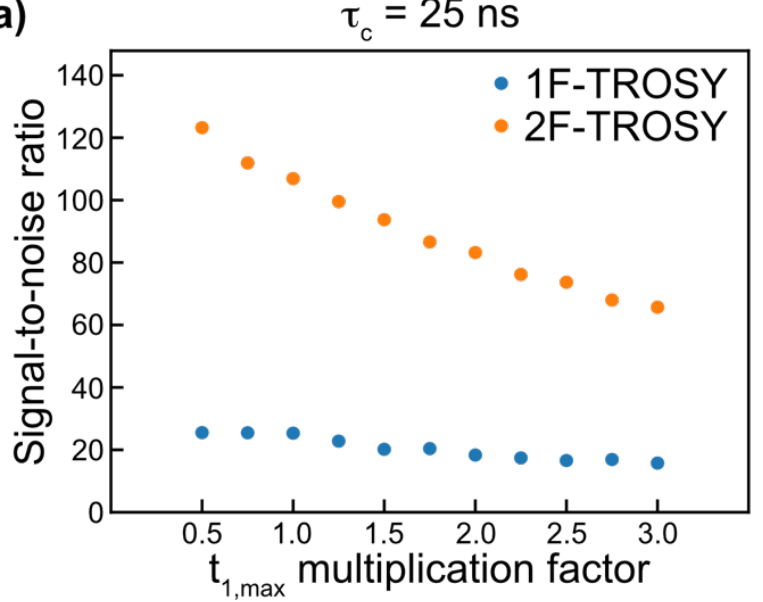

b)

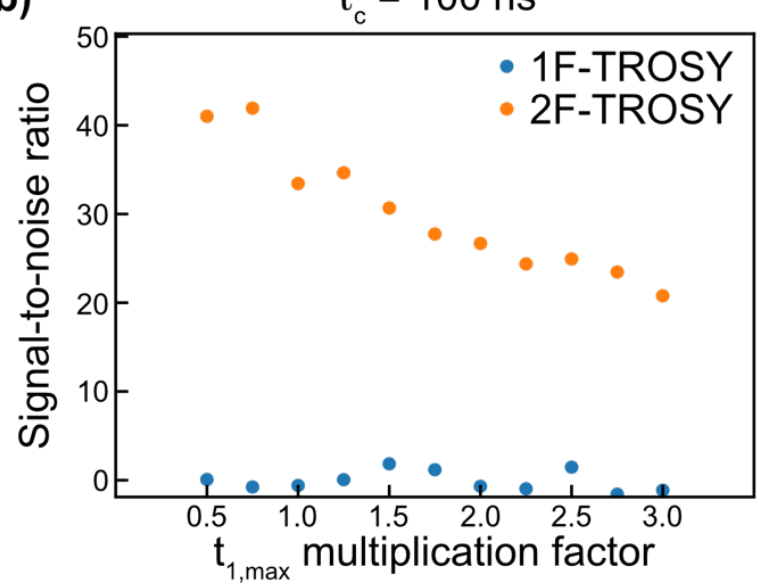

Figure 4: Expected signal-to-noise ratio (SNR) for the 1F-TROSY and 2F-TROSY experiments. The SNR was estimated for proteins with global tumbling correlation times $\tau_{c}=25 \mathrm{~ns}(\mathbf{a})$ and $\tau_{\mathrm{c}}=100 \mathrm{~ns}(\mathbf{b})$ labeled with 3F-Tyr. The value of $\mathrm{t}_{1, \max }$ is calculated as $t_{1, \max }(C)=C \times T_{2}\left({ }^{19} \mathrm{~F}\right)$ where $\mathrm{C}$ is a multiplication factor, ranging from 0.5 to 3.0 , and $\mathrm{T}_{2}\left({ }^{19} \mathrm{~F}\right)$ is the transverse relaxation time for the fluorine TROSY single-transition coherence (resp. the in-phase single-quantum coherence) in the 1FTROSY (resp. 2F-TROSY) experiment. The experimental time is the same for each simulated experiment (4.9 hours), leading to a higher number of scans for experiments with shorter $t_{1, \text { max }}$.

2F-TROSY offers increased sensitivity and resolution. We compared the expected SNR from the 1F-TROSY and 2F-TROSY experiments at their optimal magnetic field(s) by simulating the full two-dimensional spectra with Gaussian noise with scaled intensity from one field to the other (see Methods). The level of noise was chosen to approximately reproduce the SNR on MBP spectra already published ${ }^{15}$ (SNR ca. 30 for 80 scans of $700 \mu \mathrm{M}$ MBP, recycling delay of $2 \mathrm{sec}, 116$ complex points in the indirect dimension and $\mathrm{t}_{1, \max }=10 \mathrm{~ms}$, see Fig. S11, and Section 1 of the Supplementary Materials for more details). Peak intensities depend, among other parameters, on the number of points in the indirect time dimension, which is usually set to reach a desired resolution for a given spectral width $(\mathrm{SW})$. To take different options into account, we simulated the pulse sequences for different values of $t_{1, \max }$ (Fig. S12-S13). For moderate protein sizes $\left(\tau_{\mathrm{c}}=25 \mathrm{~ns}\right)$, the 2F-TROSY experiment offers better sensitivity by a 
factor ca. 5 for $\mathrm{t}_{1, \max }=\mathrm{T}_{2}\left({ }^{19} \mathrm{~F}\right)$ (Fig. 4.a). For a large system $\left(\tau_{\mathrm{c}}=100 \mathrm{~ns}\right)$, no visible peak is expected at the chosen level of noise for the 1F-TROSY experiment, while good SNR for the 2F-TROSY experiment can be achieved in similar experimental time (Fig. 4.b). Similar results are obtained for compounds with better ${ }^{13} \mathrm{C}$-TROSY efficiency (i.e. lower optimal field for carbon detection) such as the 4F-Phe (Fig. S14). In the following, we consider $\mathrm{t}_{1, \max }=1.25 \mathrm{~T}_{2}\left({ }^{19} \mathrm{~F}\right) .{ }^{32}$

a)

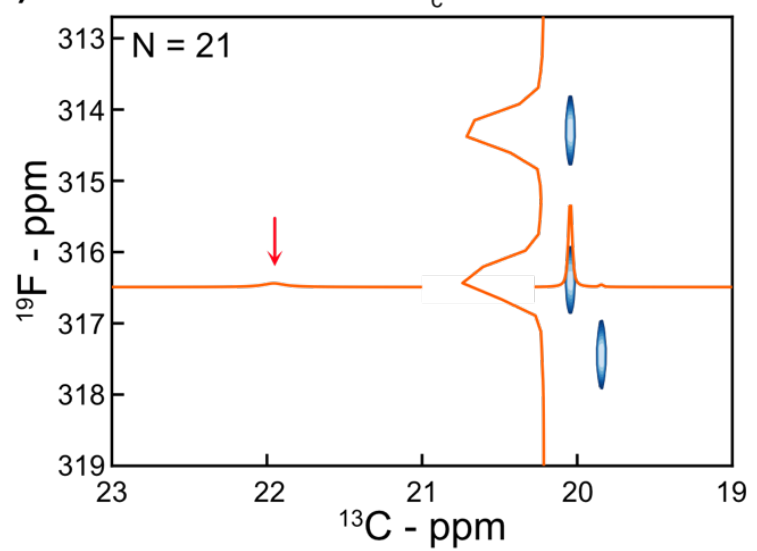

c)

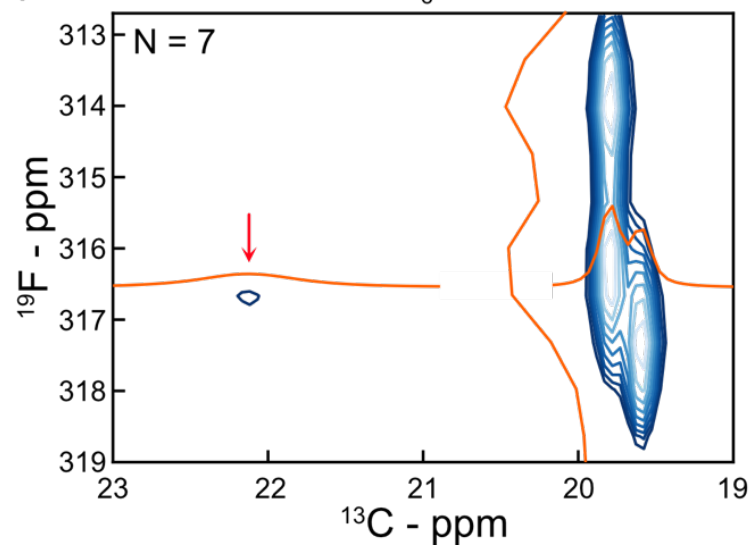

b) $\quad 2 \mathrm{~F}-\mathrm{TROSY}-\tau_{\mathrm{c}}=25 \mathrm{~ns}$

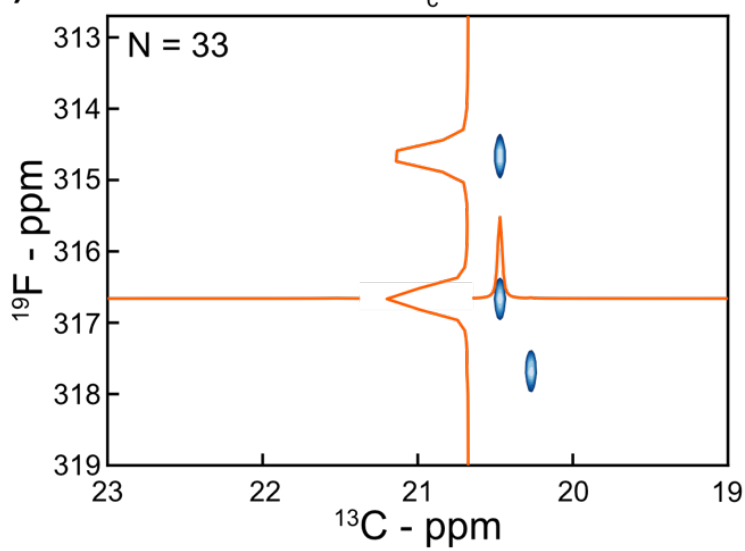

d) $\quad 2 \mathrm{~F}-\mathrm{TROSY}-\tau_{\mathrm{c}}=100 \mathrm{~ns}$

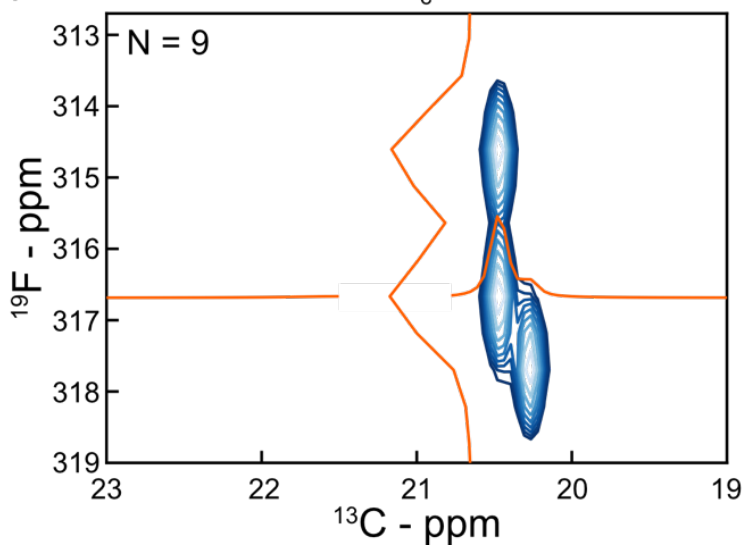

Figure 5: Simulated noise-free two-dimensional ${ }^{13} \mathrm{C}-{ }^{19} \mathrm{~F}$ TROSY spectra with 3 peaks. Spectra for the 1F-TROSY (a, c) and 2F-TROSY (b, d) experiments simulated with global tumbling correlation times $\tau_{\mathrm{c}}=25 \mathrm{~ns}(\mathbf{a}, \mathbf{b})$ and $\tau_{\mathrm{c}}=100 \mathrm{~ns}(\mathbf{c}, \mathbf{d})$ for $\mathrm{t}_{1, \max }=1.25 \mathrm{~T}_{2}\left({ }^{19} \mathrm{~F}\right)$ where $\mathrm{T}_{2}\left({ }^{19} \mathrm{~F}\right)$ is the transverse relaxation time of the coherence edited in the indirect dimension. In each spectrum, 10 contour levels are shown, starting from the maximum intensity and with a factor 1.2 between two consecutive levels. Spectra were simulated without noise. Cross-sections for one peak are shown in orange. In panels $\mathbf{a}$ and $\mathbf{b}$, the carbon anti-TROSY peak is shown with the red arrow. The number of recorded points in the indirect dimension is indicated on the top left corner of each panel.

Having two distinct magnetic centers allows one to carry the evolution of the ${ }^{19} \mathrm{~F}$ and ${ }^{13} \mathrm{C}$ TROSY coherences where their respective relaxation properties are most favorable. We have chosen conventional magnetic fields that lead to near-optimal sensitivity. The 2F-TROSY experiment not only leads to better sensitivity but also to better resolution in both dimensions as compared to the 1F-TROSY experiment (Fig. 5). The resolution in the carbon dimension would be the same for the two experiments if the detection is done at the same field. However, recording both experiments at $14.1 \mathrm{~T}$ to obtain a strong ${ }^{13} \mathrm{C}$-TROSY effect leads to sensitivity 
losses. The sub-optimal TROSY relaxation interference in the 1F-TROSY leads to large carbon linewidth in the spectra of large protein $\left(\tau_{\mathrm{c}}=100 \mathrm{~ns}\right)$. For a medium-size system $\left(\tau_{\mathrm{c}}=25 \mathrm{~ns}\right)$, the 1F-TROSY experiment provides good peak separation in spite of the broadening in the fluorine dimension, but the spectra of larger proteins $\left(\tau_{\mathrm{c}}=100 \mathrm{~ns}\right)$ show $c a .2$ ppm broad peaks. The 2F-TROSY experiment gives rise to a lower fluorine linewidth, even for large systems ( $c a$. $1 \mathrm{ppm})$. Combined with the optimal ${ }^{13} \mathrm{C}$-TROSY detection, satisfactory peak separation can be obtained in situations where peak overlap and a low SNR would prevent quantitative analysis in the 1F-TROSY pulse sequence.

\section{CONCLUSION}

We have introduced the concept of two-field transverse relaxation-optimized spectroscopy (2FTROSY). It takes advantage of the development in two-field NMR spectroscopy ${ }^{17,18}$ to increase the sensitivity in multidimensional spectra. By optimizing the magnetic field of different parts of the pulse sequence, the transverse relaxation rates of the evolving operators are decreased to their minimal values. We have illustrated the potential of this new type of pulse sequences on ${ }^{13} \mathrm{C}-{ }^{19} \mathrm{~F}$ aromatic spin pairs, where optimal magnetic fields are in a range that will be accessible in future designs of two-field NMR. Our calculations show that the investigation of aromatic residues could greatly benefit from two-field TROSY methods.

We expect a new generation of two-field NMR spectrometers to offer sufficiently high homogeneity to allow the evolution of single-quantum coherences at low field, an essential feature to obtain high-resolution spectra and develop a new toolbox of pulse sequences. We expect ${ }^{19} \mathrm{~F}$ aromatic side-chain specific labeling ${ }^{15}$ combined with $2 \mathrm{~F}-$ TROSY will open the way for the investigation of structure, dynamics and function of aromatic side chains in proteins, as well as purine and pyrimidine bases in nucleic acids. This would provide new probes for NMR of large proteins and nucleic acids. Further benefits are expected in systems with line broadening due to chemical exchange. ${ }^{18,33}$ Beyond the scope of ${ }^{13} \mathrm{C}-{ }^{19} \mathrm{~F}$, two-field NMR approaches that exploit favorable relaxation properties at low field can be adapted to the NMR investigation of a variety of nuclei with large CSAs (such as the carbonyl carbon of peptide bonds) to increase the sensitivity and resolution of NMR of large complex systems.

\section{REFERENCES}

(1) Kay, L. E.; Gardner, K. H. Solution NMR Spectroscopy beyond 25 kDa. Curr Opin Struct Biol 1997, 7, 722-731.

(2) Sprangers, R.; Kay, L. E. Quantitative Dynamics and Binding Studies of the 20S Proteasome by NMR. Nature 2007, 445 (7128), 618-622.

https://doi.org/10.1038/nature05512. 
(3) Pervushin, K.; Riek, R.; Wider, G.; Wuthrich, K. Attenuated T2 Relaxation by Mutual Cancellation of Dipole-Dipole Coupling and Chemical Shift Anisotropy Indicates an Avenue to NMR Structures of Very Large Biological Macromolecules in Solution.

Proc. Natl. Acad. Sci. 1997, 94 (23), 12366-12371.

https://doi.org/10.1073/pnas.94.23.12366.

(4) Pervushin, K.; Riek, R.; Wider, G.; Wüthrich, K. Transverse Relaxation-Optimized Spectroscopy (TROSY) for NMR Studies of Aromatic Spin Systems in ${ }^{13}$ C-Labeled Proteins. J. Am. Chem. Soc. 1998, 120 (25), 6394-6400.

https://doi.org/10.1021/ja980742g.

(5) Riek, R.; Pervushin, K.; Fernández, C.; Kainosho, M.; Wüthrich, K. [ $\left.{ }^{13} \mathrm{C},{ }^{13} \mathrm{C}\right]-$ and [

$\left.{ }^{13} \mathrm{C},{ }^{1} \mathrm{H}\right]$-TROSY in a Triple Resonance Experiment for Ribose-Base and Intrabase

Correlations in Nucleic Acids ${ }^{1}$. J. Am. Chem. Soc. 2001, 123 (4), 658-664.

https://doi.org/10.1021/ja9938276.

(6) Brutscher, B.; Boisbouvier, J.; Pardi, A.; Marion, D.; Simorre, J.-P. Improved Sensitivity and Resolution in ${ }^{1} \mathrm{H}-{ }^{13} \mathrm{C}$ NMR Experiments of RNA. J. Am. Chem. Soc. 1998, 120 (46), 11845-11851. https://doi.org/10.1021/ja9828531.

(7) Tugarinov, V.; Hwang, P. M.; Ollerenshaw, J. E.; Kay, L. E. Cross-Correlated Relaxation Enhanced ${ }^{1} \mathrm{H}-{ }^{13} \mathrm{C}$ NMR Spectroscopy of Methyl Groups in Very High Molecular Weight Proteins and Protein Complexes. J. Am. Chem. Soc. 2003, 125 (34), 10420-10428. https://doi.org/10.1021/ja030153x.

(8) Korzhnev, D. M.; Kloiber, K.; Kanelis, V.; Tugarinov, V.; Kay, L. E. Probing Slow Dynamics in High Molecular Weight Proteins by Methyl-TROSY NMR Spectroscopy: Application to a 723-Residue Enzyme. J. Am. Chem. Soc. 2004, 126 (12), 3964-3973. https://doi.org/10.1021/ja039587i.

(9) Abramov, G.; Velyvis, A.; Rennella, E.; Wong, L. E.; Kay, L. E. A Methyl-TROSY Approach for NMR Studies of High-Molecular-Weight DNA with Application to the Nucleosome Core Particle. Proc. Natl. Acad. Sci. 2020, 117 (23), 12836-12846. https://doi.org/10.1073/pnas.2004317117.

(10) Shimizu, H. Theory of the Dependence of Nuclear Magnetic Relaxation on the Absolute Sign of Spin-Spin Coupling Constant. J. Chem. Phys. 1964, 40 (11), 33573364. https://doi.org/10.1063/1.1725007.

(11) Goldman, M. Interference Effects in the Relaxation of a Pair of Unlike Spin-1/2 Nuclei. 1984, 60, 437-452. https://doi.org/0022-236418.

(12) Wimperis, S.; Bodenhausen, G. Relaxation-Allowed Cross-Peaks in Two-Dimensional N.M.R. Correlation Spectroscopy. Mol. Phys. 1989, 66 (5), 897-919. https://doi.org/10.1080/00268978900100621.

(13) Bolik-Coulon, N.; Cousin, S. F.; Kadeřávek, P.; Dumez, J.-N.; Ferrage, F. Understanding the Methyl-TROSY Effect over a Wide Range of Magnetic Fields. $J$. Chem. Phys. 2019, 150 (22), 224202. https://doi.org/10.1063/1.5095757.

(14) Takeuchi, K.; Arthanari, H.; Wagner, G. Perspective: Revisiting the Field Dependence of TROSY Sensitivity. J. Biomol. NMR 2016, 66 (4), 221-225. https://doi.org/10.1007/s10858-016-0075-4.

(15) Boeszoermenyi, A.; Chhabra, S.; Dubey, A.; Radeva, D. L.; Burdzhiev, N. T.; Chanev, C. D.; Petrov, O. I.; Gelev, V. M.; Zhang, M.; Anklin, C.; Kovacs, H.; Wagner, G.; Kuprov, I.; Takeuchi, K.; Arthanari, H. Aromatic 19F-13C TROSY: A BackgroundFree Approach to Probe Biomolecular Structure, Function, and Dynamics. Nat. Methods 2019, 16 (4), 333-340. https://doi.org/10.1038/s41592-019-0334-x.

(16) Kreutz, C.; Nußbaumer, F.; Plangger, R.; Roeck, M. Aromatic 19F-13C TROSY [19F, 13C] Pyrimidine Labeling for NMR Spectroscopy of RNA. Angew. Chem. Int. Ed. 2020. https://doi.org/10.1002/anie.202006577. 
(17) Cousin, S. F.; Charlier, C.; Kadeřávek, P.; Marquardsen, T.; Tyburn, J.-M.; Bovier, P.A.; Ulzega, S.; Speck, T.; Wilhelm, D.; Engelke, F.; Maas, W.; Sakellariou, D.; Bodenhausen, G.; Pelupessy, P.; Ferrage, F. High-Resolution Two-Field Nuclear Magnetic Resonance Spectroscopy. Phys. Chem. Chem. Phys. 2016, 18 (48), 3318733194. https://doi.org/10.1039/C6CP05422F.

(18) Cousin, S. F.; Kadeřávek, P.; Haddou, B.; Charlier, C.; Marquardsen, T.; Tyburn, J.M.; Bovier, P.-A.; Engelke, F.; Maas, W.; Bodenhausen, G.; Pelupessy, P.; Ferrage, F. Recovering Invisible Signals by Two-Field NMR Spectroscopy. Angew. Chem. Int. Ed. 2016, 55 (34), 9886-9889. https://doi.org/10.1002/anie.201602978.

(19) Kadeř́vek, P.; Strouk, L.; Cousin, S. F.; Charlier, C.; Bodenhausen, G.; Marquardsen, T.; Tyburn, J.-M.; Bovier, P.-A.; Engelke, F.; Maas, W.; Ferrage, F. Full Correlations across Broad NMR Spectra by Two-Field Total Correlation Spectroscopy. ChemPhysChem 2017, 18 (19), 2772-2776. https://doi.org/10.1002/cphc.201700369.

(20) Kadeřávek, P.; Bolik-Coulon, N.; Cousin, S. F.; Marquardsen, T.; Tyburn, J.-M.; Dumez, J.-N.; Ferrage, F. Protein Dynamics from Accurate Low-Field Site-Specific Longitudinal and Transverse Nuclear Spin Relaxation. J. Phys. Chem. Lett. 2019, 10 (19), 5917-5922. https://doi.org/10.1021/acs.jpclett.9b02233.

(21) Jaseňáková, Z.; Zapletal, V.; Padrta, P.; Zachrdla, M.; Bolik-Coulon, N.; Marquardsen, T.; Tyburn, J.-M.; Žídek, L.; Ferrage, F.; Kadeřávek, P. Boosting the Resolution of Low-Field 15N Relaxation Experiments on Intrinsically Disordered Proteins with Triple-Resonance NMR. J. Biomol. NMR 2020, 74 (2-3), 139-145. https://doi.org/10.1007/s10858-019-00298-6.

(22) Bolik-Coulon, N.; Kadeřávek, P.; Pelupessy, P.; Dumez, J.-N.; Ferrage, F.; Cousin, S. F. Theoretical and Computational Framework for the Analysis of the Relaxation Properties of Arbitrary Spin Systems. Application to High-Resolution Relaxometry. $J$. Magn. Reson. 2020, 313, 106718. https://doi.org/10.1016/j.jmr.2020.106718.

(23) Lipari, G.; Szabo, A. Model-Free Approach to the Interpretation of Nuclear Magnetic Resonance Relaxation in Macromolecules. 1. Theory and Range of Validity. J. Am. Chem. Soc. 1982, 104 (17), 4546-4559. https://doi.org/10.1021/ja00381a009.

(24) Allard, P.; Helgstrand, M.; Härd, T. The Complete Homogeneous Master Equation for a Heteronuclear Two-Spin System in the Basis of Cartesian Product Operators. $J$. Magn. Reson. 1998, 134 (1), 7-16. https://doi.org/10.1006/jmre.1998.1509.

(25) van der Walt, S.; Colbert, S. C.; Varoquaux, G. The NumPy Array: A Structure for Efficient Numerical Computation. Comput. Sci. Eng. 2011, 13 (2), 22-30. https://doi.org/10.1109/MCSE.2011.37.

(26) SciPy 1.0 Contributors; Virtanen, P.; Gommers, R.; Oliphant, T. E.; Haberland, M.; Reddy, T.; Cournapeau, D.; Burovski, E.; Peterson, P.; Weckesser, W.; Bright, J.; van der Walt, S. J.; Brett, M.; Wilson, J.; Millman, K. J.; Mayorov, N.; Nelson, A. R. J.; Jones, E.; Kern, R.; Larson, E.; Carey, C. J.; Polat, İ.; Feng, Y.; Moore, E. W.; VanderPlas, J.; Laxalde, D.; Perktold, J.; Cimrman, R.; Henriksen, I.; Quintero, E. A.; Harris, C. R.; Archibald, A. M.; Ribeiro, A. H.; Pedregosa, F.; van Mulbregt, P. SciPy 1.0: Fundamental Algorithms for Scientific Computing in Python. Nat. Methods 2020, 17 (3), 261-272. https://doi.org/10.1038/s41592-019-0686-2.

(27) Helmus, J. J.; Jaroniec, C. P. Nmrglue: An Open Source Python Package for the Analysis of Multidimensional NMR Data. J. Biomol. NMR 2013, 55 (4), 355-367. https://doi.org/10.1007/s10858-013-9718-x.

(28) Levitt, M. H.; Bari, L. D. The Homogeneous Master Equation and the Manipulation of Relaxation Networks. $16(1), 21$. 
(29) Ottiger, M.; Bax, A. Determination of Relative $\mathrm{N}-\mathrm{H}^{\mathrm{N}}, \mathrm{N}-\mathrm{C}^{6}, \mathrm{C}^{\alpha}-\mathrm{C}^{6}$, and $\mathrm{C}^{\alpha}-\mathrm{H}^{\alpha}$ Effective Bond Lengths in a Protein by NMR in a Dilute Liquid Crystalline Phase. $J$. Am. Chem. Soc. 1998, 120 (47), 12334-12341. https://doi.org/10.1021/ja9826791.

(30) Meissner, A.; Duus, J. Ø.; Sørensen, O. W. Integration of Spin-State-Selective Excitation into 2D NMR Correlation Experiments with Heteronuclear ZQ/2Q $\pi$ Rotations for 1JXH-Resolved E.COSY-Type Measurement of Heteronuclear Coupling Constants in Proteins. J. Biomol. NMR. 1997, 10, 89.

(31) Pervushin, K.; Wider, G.; Wüthrich, K. Single Transition-to-Single Transition Polarization Transfer (ST2-PT) in [15N,1H]-TROSY. J. Biomol. NMR. 1998, 12, 345.

(32) Rovnyak, D. The Past, Present, and Future of $1.26 T_{2}$. Concepts Magn. Reson. Part A 2018, $47 A$ (2), e21473. https://doi.org/10.1002/cmr.a.21473.

(33) Arntson, K. E.; Pomerantz, W. C. K. Protein-Observed Fluorine NMR: A Bioorthogonal Approach for Small Molecule Discovery: Miniperspective. J. Med. Chem. 2016, 59 (11), 5158-5171. https://doi.org/10.1021/acs.jmedchem.5b01447. 


\section{Two-Field Transverse Relaxation-Optimized Spectroscopy for the Study of Large Biomolecules - an in silico investigation}

Nicolas Bolik-Coulon ${ }^{1}$, Philippe Pelupessy ${ }^{1}$, Guillaume Bouvignies $^{1}$, and Fabien Ferrage ${ }^{1}$

${ }^{1}$ Laboratoire des Biomolécules, LBM, Département de Chimie, École Normale

Supérieure, PSL University, Sorbonne Université, CNRS, 75005 Paris, France

\section{Supplementary Materials}

\section{Contents}

1 Method details $\quad 2$

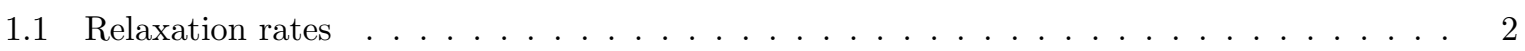

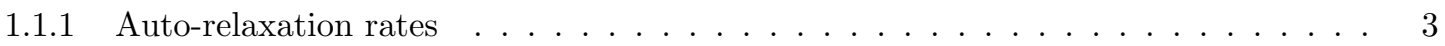

1.1.2 Cross-relaxation rates . . . . . . . . . . . . . . . . . 4

1.2 Simulation, processing and analysis of a spectra . . . . . . . . . . . . . 5

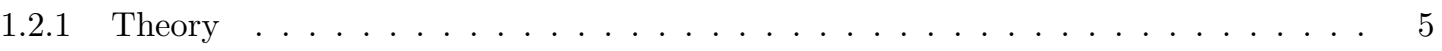

1.2 .2 Acquisition details . . . . . . . . . . . . . . . . 6

1.2.3 Optimization of the recycling delays and simulation of dummy scans . . . . . . . . 7

1.2.4 Extracting peak heights from noise-free spectra . . . . . . . . . . . . . . 7

1.2.5 Processing of the spectra .................... 8

1.3 Noise simulation and signal-to-noise assessment . . . . . . . . . . . . . . . 8

2 Product operator analysis of the pulse sequences $r$

2.1 Single-field TROSY experiment . . . . . . . . . . . . . . . . . . . . 10

2.2 Two-field TROSY experiment . . . . . . . . . . . . . . . . . . . . 11

3 Additional Figures $r$ 


\section{Method details}

\subsection{Relaxation rates}

The basis formed by a ${ }^{13} \mathrm{C}-{ }^{19} \mathrm{~F}$ contains 16 terms:

$$
\left\{\hat{E}, \hat{\mathrm{F}}_{\mathrm{x}}, \hat{\mathrm{F}}_{\mathrm{y}}, \hat{\mathrm{F}}_{\mathrm{z}}, \hat{\mathrm{C}}_{\mathrm{x}}, \hat{\mathrm{C}}_{\mathrm{y}}, \hat{\mathrm{C}}_{\mathrm{z}}, 2 \hat{\mathrm{F}}_{\mathrm{x}} \hat{\mathrm{C}}_{\mathrm{z}}, 2 \hat{\mathrm{F}}_{\mathrm{y}} \hat{\mathrm{C}}_{\mathrm{z}}, 2 \hat{\mathrm{F}}_{\mathrm{z}} \hat{\mathrm{C}}_{\mathrm{x}}, 2 \hat{\mathrm{F}}_{\mathrm{z}} \hat{\mathrm{C}}_{\mathrm{y}}, 2 \hat{\mathrm{F}}_{\mathrm{x}} \hat{\mathrm{C}}_{\mathrm{x}}, 2 \hat{\mathrm{F}}_{\mathrm{x}} \hat{\mathrm{C}}_{\mathrm{y}}, 2 \hat{\mathrm{F}}_{\mathrm{y}} \hat{\mathrm{C}}_{\mathrm{x}}, 2 \hat{\mathrm{F}}_{\mathrm{y}} \hat{\mathrm{C}}_{\mathrm{y}}, 2 \hat{\mathrm{F}}_{\mathrm{z}} \hat{\mathrm{C}}_{\mathrm{z}}\right\}
$$

We report here relaxation rates of each of these operators and cross-relaxation between them, as calculated using RedKite [1]. They are also shown in Fig. S1.

In the following, $d_{\mathrm{CF}}=\frac{-\mu_{0} \hbar \gamma_{\mathrm{C}} \gamma_{\mathrm{F}}}{4 \pi r_{\mathrm{CF}}^{3}}$ is the dipolar coefficient for the interaction between the carbon and fluorine, $\gamma_{\mathrm{C}}$ and $\gamma_{\mathrm{F}}$ are their respective gyromagnetic ratios, $r_{\mathrm{CF}}$ is the internuclear distance, $\mu_{0}$ is the permability of free space and $\hbar$ the Planck's constant devided by $2 \pi$. The chemical shift anisotropy (CSA) tensors of the carbon and fluorine are asymmetric with $\sigma_{\|, i}$ being the longitudinal component aligned in the direction of the CF bond with angle reported in Ref. [2] and Table S1 and $\sigma_{\perp, i}$ the orthogonal component perpendicular to the ring plane.

Table S1: CSA tensor parameters as reported in Ref. [2] and TROSY operators. The parallel components of the CSAs lay in the aromatic ring plane at an angle $\alpha$ with respect to the $\mathrm{C}-\mathrm{F}$ bond, while the perpendicular components are oriented perpendicular to this plane.

\begin{tabular}{|c|c|c|c|c|c|c|c|c|c|}
\hline \multirow{2}{*}{\multicolumn{2}{|c|}{ Molecule }} & \multicolumn{2}{|c|}{${ }^{19} \mathrm{~F}-\mathrm{CSA}, \mathrm{ppm}$} & \multicolumn{2}{|c|}{${ }^{13} \mathrm{C}-\mathrm{CSA}, \mathrm{ppm}$} & \multicolumn{2}{|c|}{$\alpha,{ }^{\circ}$} & \multicolumn{2}{|c|}{ TROSY operator } \\
\hline & & $\sigma_{\|}^{F}$ & $\sigma_{\perp}^{F}$ & $\sigma_{\|}^{C}$ & $\sigma_{\perp}^{C}$ & ${ }^{19} \mathrm{~F}$ & ${ }^{13} \mathrm{C}$ & Fluorine & Carbon \\
\hline 3F-Tyr & 3-fluorotyrosine & 43 & 157 & -75 & 54 & 17 & 16 & $\hat{\mathrm{F}}^{+}-2 \hat{\mathrm{F}}^{+} \hat{\mathrm{C}}_{z}$ & $\hat{\mathrm{C}}^{+}-2 \hat{\mathrm{F}}_{z} \hat{\mathrm{C}}^{+}$ \\
\hline 4F-Phe & 4-fluorophenylalanine & 81 & 139 & -111 & 51 & 0 & 0 & $\hat{\mathrm{F}}^{+}+2 \hat{\mathrm{F}}^{+} \hat{\mathrm{C}}_{z}$ & $\hat{\mathrm{C}}^{+}-2 \hat{\mathrm{F}}_{z} \hat{\mathrm{C}}^{+}$ \\
\hline
\end{tabular}

We used the following spectral density function evaluated at frequency $\omega$ when describing the dynamics of the aromatic ${ }^{13} \mathrm{C}-{ }^{19} \mathrm{~F}$ group:

$$
\mathcal{J}_{\vec{A}, \vec{B}}(\omega)=\frac{2}{5} \mathcal{P}_{2}\left(\cos \left(\theta_{\vec{A}, \vec{B}}\right)\right)\left(S^{2} \frac{\tau_{c}}{1+\left(\omega \tau_{c}\right)^{2}}+\left(1-S^{2}\right) \frac{\tau_{\text {int }}^{\prime}}{1+\left(\omega \tau_{\text {int }}^{\prime}\right)^{2}}\right),
$$

with $\vec{A}$ and $\vec{B}$ are the vectors along the interactions $\mathbf{A}$ and $\mathbf{B}$ (in the case of auto-correlation, $\mathbf{A}=\mathbf{B}$ ), $\theta_{\vec{A}, \vec{B}}$ is the angle between interactions $\mathbf{A}$ and $\mathbf{B}, \mathcal{P}_{2}$ is the second order Legendre polynomial, $S^{2}$ is the order parameter of the CF group, $\tau_{c}$ is the global tumbling correlation time of the protein and $\tau_{\text {int }}^{\prime-1}=\tau_{\text {int }}^{-1}+\tau_{c}^{-1}$ with $\tau_{\text {int }}$ an internal correlation time to describe the local motions of the CF bond. Here, we set $S^{2}=0.8$ and $\tau_{\text {int }}=100$ ps. Notations reported in Table S2 will be used in the expressions of relaxation rates. Table $\mathrm{S} 2$ also reports the value of the angles $\theta_{\vec{A}, \vec{B}}$. 
Table S2: Spectral density functions used in the expressions of relaxation rates for a ${ }^{13} \mathrm{C}-{ }^{19} \mathrm{~F}$ group and associated value of $\theta_{\vec{A}, \vec{B}}$. The CSA auto-correlation spectral density function is equal to $\mathcal{J}_{\mathrm{CF}}$. DD: Dipole-Dipole. CSA: Chemical Shift Anisotropy. $\alpha_{\mathrm{i}}$ : angle of the principal CSA tensor with the z-axis for nuclei $i$ (TableS1).

\begin{tabular}{|cccc|}
\hline Notation & Correlation & Interaction & $\theta_{\vec{A}, \vec{B}}$ \\
\hline $\mathcal{J}_{\mathrm{CF}}$ & auto-correlation C-F & $\mathrm{DD}$ & 0 \\
$\mathcal{J}_{\text {csa }}$ & cross-correlation between homonuclear components of CSA tensor & $\sigma_{\|, i} / \sigma_{\perp, i}$ & $\pi / 2$ \\
$\mathcal{J}_{\mathrm{CFF}}$ & cross-correlation between the ${ }^{19} \mathrm{~F}$ longitudinal CSA and C-F & $\mathrm{DD} / \sigma_{\|, F}$ & $\alpha_{\mathrm{F}}$ \\
$\mathcal{J}_{\mathrm{CFC}}$ & cross-correlation between the ${ }^{13} \mathrm{C}$ longitudinal CSA and C-F & $\mathrm{DD} / \sigma_{\|, C}$ & $\alpha_{\mathrm{C}}$ \\
$\mathcal{J}_{\mathrm{CF} \sigma_{\perp}}$ & cross-correlation between the orthogonal CSA and C-F & $\mathrm{DD} / \sigma_{\perp, i}$ & $\pi / 2$ \\
$\mathcal{J}_{\sigma_{\|}^{F}, \sigma_{\|}^{C}}$ & cross-correlation between heteronuclear components of CSA tensor & $\sigma_{\|, F} / \sigma_{\|, C}$ & $\alpha_{\mathrm{F}}-\alpha_{\mathrm{C}}$ \\
$\mathcal{J}_{\sigma_{\|}^{F}, \sigma_{\perp}^{C}}$ & cross-correlation between heteronuclear components of CSA tensor & $\sigma_{\|, F} / \sigma_{\perp, C}$ & $\pi / 2$ \\
$\mathcal{J}_{\sigma_{\perp}^{F}, \sigma_{\|}^{C}}$ & cross-correlation between heteronuclear components of CSA tensor & $\sigma_{\perp, F} / \sigma_{\|, C}$ & $\pi / 2$ \\
$\mathcal{J}_{\sigma_{\perp}^{F}, \sigma_{\perp}^{C}}$ & cross-correlation between heteronuclear components of CSA tensor & $\sigma_{\perp, F} / \sigma_{\perp, C}$ & 0 \\
\hline
\end{tabular}

\subsubsection{Auto-relaxation rates}

$$
\begin{aligned}
\mathcal{R}_{2}(\mathrm{~F})= & \mathcal{R}\left(\hat{\mathrm{F}}_{\mathrm{x}}\right)=\mathcal{R}\left(\hat{\mathrm{F}}_{\mathrm{y}}\right) \\
= & \frac{d_{\mathrm{CF}}^{2}}{8}\left(4 \mathcal{J}_{\mathrm{CF}}(0)+\mathcal{J}_{\mathrm{CF}}\left(\omega_{\mathrm{F}}-\omega_{\mathrm{C}}\right)+6 \mathcal{J}_{\mathrm{CF}}\left(\omega_{\mathrm{C}}\right)+3 \mathcal{J}_{\mathrm{CF}}\left(\omega_{\mathrm{F}}\right)+6 \mathcal{J}_{\mathrm{CF}}\left(\omega_{\mathrm{F}}+\omega_{\mathrm{C}}\right)\right) \\
& +\frac{\omega_{\mathrm{F}}^{2}}{18}\left(\left(\sigma_{\|, F}^{2}+\sigma_{\perp, F}^{2}\right)\left(4 \mathcal{J}_{\mathrm{CF}}(0)+3 \mathcal{J}_{\mathrm{CF}}\left(\omega_{\mathrm{F}}\right)\right)+2 \sigma_{\|, F} \sigma_{\perp, F}\left(4 \mathcal{J}_{\mathrm{csa}}(0)+3 \mathcal{J}_{\mathrm{csa}}\left(\omega_{\mathrm{F}}\right)\right)\right) \\
\mathcal{R}_{1}(\mathrm{~F})= & \mathcal{R}\left(\hat{\mathrm{F}}_{\mathrm{z}}\right) \\
= & \frac{d_{\mathrm{CF}}^{2}}{4}\left(\mathcal{J}_{\mathrm{CF}}\left(\omega_{\mathrm{F}}-\omega_{\mathrm{C}}\right)+3 \mathcal{J}_{\mathrm{CF}}\left(\omega_{\mathrm{F}}\right)+6 \mathcal{J}_{\mathrm{CF}}\left(\omega_{\mathrm{F}}+\omega_{\mathrm{C}}\right)\right) \\
& +\frac{\omega_{\mathrm{F}}^{2}}{3}\left(\left(\sigma_{\|, F}^{2}+\sigma_{\perp, F}^{2}\right) \mathcal{J}_{\mathrm{CF}}\left(\omega_{\mathrm{F}}\right)+2 \sigma_{\|, F} \sigma_{\perp, F} \mathcal{J}_{\mathrm{csa}}\left(\omega_{\mathrm{F}}\right)\right) \\
\mathcal{R}_{2}(\mathrm{C})= & \mathcal{R}\left(\hat{\mathrm{C}}_{\mathrm{x}}\right)=\mathcal{R}\left(\hat{\mathrm{C}}_{\mathrm{y}}\right) \\
= & \frac{d_{\mathrm{CF}}^{2}}{8}\left(4 \mathcal{J}_{\mathrm{CF}}(0)+\mathcal{J}_{\mathrm{CF}}\left(\omega_{\mathrm{F}}-\omega_{\mathrm{C}}\right)+6 \mathcal{J}_{\mathrm{CF}}\left(\omega_{\mathrm{F}}\right)+3 \mathcal{J}_{\mathrm{CF}}\left(\omega_{\mathrm{C}}\right)+6 \mathcal{J}_{\mathrm{CF}}\left(\omega_{\mathrm{F}}+\omega_{\mathrm{C}}\right)\right) \\
& +\frac{\omega_{\mathrm{C}}^{2}}{18}\left(\left(\sigma_{\|, C}^{2}+\sigma_{\perp, C}^{2}\right)\left(4 \mathcal{J}_{\mathrm{CF}}(0)+3 \mathcal{J}_{\mathrm{CF}}\left(\omega_{\mathrm{C}}\right)\right)+2 \sigma_{\|, C} \sigma_{\perp, C}\left(4 \mathcal{J}_{\mathrm{cs}}(0)+3 \mathcal{J}_{\mathrm{csa}}\left(\omega_{\mathrm{C}}\right)\right)\right) \\
\mathcal{R}_{1}(\mathrm{C})= & \mathcal{R}\left(\hat{\mathrm{C}}_{\mathrm{z}}\right) \\
= & \frac{d_{\mathrm{CF}}^{2}}{4}\left(\mathcal{J}_{\mathrm{CF}}\left(\omega_{\mathrm{F}}-\omega_{\mathrm{C}}\right)+3 \mathcal{J}_{\mathrm{CF}}\left(\omega_{\mathrm{C}}\right)+6 \mathcal{J}_{\mathrm{CF}}\left(\omega_{\mathrm{F}}+\omega_{\mathrm{C}}\right)\right) \\
& +\frac{\omega_{\mathrm{C}}^{2}}{3}\left(\left(\sigma_{\|, C}^{2}+\sigma_{\perp, C}^{2}\right) \mathcal{J}_{\mathrm{CF}}\left(\omega_{\mathrm{C}}\right)+2 \sigma_{\|, C} \sigma_{\perp, C} \mathcal{J}_{\mathrm{csa}}\left(\omega_{\mathrm{C}}\right)\right)
\end{aligned}
$$




$$
\begin{aligned}
\rho_{F}= & \mathcal{R}\left(2 \hat{\mathrm{F}}_{\mathrm{x}} \hat{\mathrm{C}}_{\mathrm{z}}\right)=\mathcal{R}\left(2 \hat{\mathrm{F}}_{\mathrm{y}} \hat{\mathrm{C}}_{\mathrm{z}}\right) \\
= & \frac{d_{\mathrm{CF}}^{2}}{8}\left(4 \mathcal{J}_{\mathrm{CF}}(0)+\mathcal{J}_{\mathrm{CF}}\left(\omega_{\mathrm{F}}-\omega_{\mathrm{C}}\right)+3 \mathcal{J}_{\mathrm{CF}}\left(\omega_{\mathrm{F}}\right)+6 \mathcal{J}_{\mathrm{CF}}\left(\omega_{\mathrm{F}}+\omega_{\mathrm{C}}\right)\right) \\
& +\frac{\omega_{\mathrm{F}}^{2}}{18}\left(\left(\sigma_{\|, F}^{2}+\sigma_{\perp, F}^{2}\right)\left(4 \mathcal{J}_{\mathrm{CF}}(0)+3 \mathcal{J}_{\mathrm{CF}}\left(\omega_{\mathrm{F}}\right)\right)+2 \sigma_{\|, F} \sigma_{\perp, F}\left(4 \mathcal{J}_{\mathrm{csa}}(0)+3 \mathcal{J}_{\mathrm{csa}}\left(\omega_{\mathrm{F}}\right)\right)\right) \\
& +\frac{\omega_{\mathrm{C}}^{2}}{3}\left(\left(\sigma_{\|, C}^{2}+\sigma_{\perp, C}^{2}\right) \mathcal{J}_{\mathrm{csa}}(0)+2 \sigma_{\|, C} \sigma_{\perp, C} \mathcal{J}_{\mathrm{csa}}(0)\right) \\
\rho_{C}= & \mathcal{R}\left(2 \hat{\mathrm{F}}_{\mathrm{z}} \hat{\mathrm{C}}_{\mathrm{x}}\right)=\mathcal{R}\left(2 \hat{\mathrm{F}}_{\mathrm{z}} \hat{\mathrm{C}}_{\mathrm{y}}\right) \\
= & \frac{d_{\mathrm{CF}}^{2}}{8}\left(4 \mathcal{J}_{\mathrm{CF}}(0)+\mathcal{J}_{\mathrm{CF}}\left(\omega_{\mathrm{F}}-\omega_{\mathrm{C}}\right)+3 \mathcal{J}_{\mathrm{CF}}\left(\omega_{\mathrm{C}}\right)+6 \mathcal{J}_{\mathrm{CF}}\left(\omega_{\mathrm{F}}+\omega_{\mathrm{C}}\right)\right) \\
& +\frac{\omega_{\mathrm{C}}^{2}}{18}\left(\left(\sigma_{\|, C}^{2}+\sigma_{\perp, C}^{2}\right)\left(4 \mathcal{J}_{\mathrm{CF}}(0)+3 \mathcal{J}_{\mathrm{CF}}\left(\omega_{\mathrm{C}}\right)\right)+2 \sigma_{\|, C} \sigma_{\perp, C}\left(4 \mathcal{J}_{\mathrm{csa}}(0)+3 \mathcal{J}_{\mathrm{csa}}\left(\omega_{\mathrm{C}}\right)\right)\right) \\
& +\frac{\omega_{\mathrm{F}}^{2}}{3}\left(\left(\sigma_{\|, F}^{2}+\sigma_{\perp, F}^{2}\right) \mathcal{J}_{\mathrm{csa}}(0)+2 \sigma_{\|, F} \sigma_{\perp, F} \mathcal{J}_{\mathrm{csa}}(0)\right) \\
\lambda_{m q}= & \mathcal{R}\left(2 \hat{\mathrm{F}}_{\mathrm{x}} \hat{\mathrm{C}}_{\mathrm{x}}\right)=\mathcal{R}\left(2 \hat{\mathrm{F}}_{\mathrm{y}} \hat{\mathrm{C}}_{\mathrm{y}}\right)=\mathcal{R}\left(2 \hat{\mathrm{F}}_{\mathrm{y}} \hat{\mathrm{C}}_{\mathrm{x}}\right)=\mathcal{R}\left(2 \hat{\mathrm{F}}_{\mathrm{x}} \hat{\mathrm{C}}_{\mathrm{y}}\right) \\
= & \frac{d_{\mathrm{CF}}^{2}}{8}\left(3 \mathcal{J}_{\mathrm{CF}}\left(\omega_{\mathrm{C}}\right)+\mathcal{J}_{\mathrm{CF}}\left(\omega_{\mathrm{F}}-\omega_{\mathrm{C}}\right)+3 \mathcal{J}_{\mathrm{CF}}\left(\omega_{\mathrm{F}}\right)+6 \mathcal{J}_{\mathrm{CF}}\left(\omega_{\mathrm{F}}+\omega_{\mathrm{F}}\right)\right) \\
& +\frac{\omega_{\mathrm{F}}^{2}}{18}\left(\left(\sigma_{\|, F}^{2}+\sigma_{\perp, F}^{2}\right)\left(4 \mathcal{J}_{\mathrm{CF}}(0)+3 \mathcal{J}_{\mathrm{CF}}\left(\omega_{\mathrm{F}}\right)\right)+2 \sigma_{\|, F} \sigma_{\perp, F}\left(4 \mathcal{J}_{\mathrm{csa}}(0)+3 \mathcal{J}_{\mathrm{csa}}\left(\omega_{\mathrm{F}}\right)\right)\right) \\
& +\frac{\omega_{\mathrm{C}}^{2}}{18}\left(\left(\sigma_{\|, C}^{2}+\sigma_{\perp, C}^{2}\right)\left(4 \mathcal{J}_{\mathrm{CF}}(0)+3 \mathcal{J}_{\mathrm{CF}}\left(\omega_{\mathrm{C}}\right)\right)+2 \sigma_{\|, C} \sigma_{\perp, C}\left(4 \mathcal{J}_{\mathrm{csa}}(0)+3 \mathcal{J}_{\mathrm{csa}}\left(\omega_{\mathrm{C}}\right)\right)\right) \\
\mathcal{R}_{C F}= & \mathcal{R}\left(2 \hat{\mathrm{F}}_{\mathrm{z}} \hat{\mathrm{C}}_{\mathrm{z}}\right) \\
= & \frac{3 d_{\mathrm{CF}}^{2}}{4}\left(\mathcal{J}_{\mathrm{CF}}\left(\omega_{\mathrm{C}}\right)+\mathcal{J}_{\mathrm{CF}}\left(\omega_{\mathrm{F}}\right)\right) \\
& +\frac{\omega_{\mathrm{F}}^{2}}{3}\left(\left(\sigma_{\|, F}^{2}+\sigma_{\perp, F}^{2}\right) \mathcal{J}_{\mathrm{CF}}\left(\omega_{\mathrm{F}}\right)+\sigma_{\|, F} \sigma_{\perp, F} \mathcal{J}_{\mathrm{csa}}\left(\omega_{\mathrm{F}}\right)\right) \\
& +\frac{\omega_{\mathrm{C}}^{2}}{3}\left(\left(\sigma_{\|, C}^{2}+\sigma_{\perp, C}^{2}\right) \mathcal{J}_{\mathrm{CF}}\left(\omega_{\mathrm{C}}\right)+\sigma_{\|, C} \sigma_{\perp, C} \mathcal{J}_{\mathrm{csa}}\left(\omega_{\mathrm{C}}\right)\right) \\
&
\end{aligned}
$$

\subsubsection{Cross-relaxation rates}

$$
\begin{aligned}
\sigma= & \mathcal{R}\left(\hat{\mathrm{F}}_{\mathrm{z}} \leftrightarrow \hat{\mathrm{C}}_{\mathrm{z}}\right) \\
= & \frac{d_{\mathrm{CF}}^{2}}{4}\left(6 \mathcal{J}_{\mathrm{CF}}\left(\omega_{\mathrm{F}}+\omega_{\mathrm{C}}\right)-\mathcal{J}_{\mathrm{CF}}\left(\omega_{\mathrm{F}}-\omega_{\mathrm{C}}\right)\right) \\
\delta_{F}= & \mathcal{R}\left(\hat{\mathrm{F}}_{\mathrm{z}} \leftrightarrow 2 \hat{\mathrm{F}}_{\mathrm{z}} \hat{\mathrm{C}}_{\mathrm{z}}\right) \\
= & d_{\mathrm{CF}} \omega_{\mathrm{F}}\left(\sigma_{\|, F} \mathcal{J}_{\mathrm{CFF}_{\|}}\left(\omega_{\mathrm{F}}\right)+\sigma_{\perp, F} \mathcal{J}_{\mathrm{CF} \sigma_{\perp}}\left(\omega_{\mathrm{F}}\right)\right) \\
\delta_{C}= & \mathcal{R}\left(\hat{\mathrm{C}}_{\mathrm{z}} \leftrightarrow 2 \hat{\mathrm{F}}_{\mathrm{z}} \hat{\mathrm{C}}_{\mathrm{z}}\right) \\
= & d_{\mathrm{CF}} \omega_{\mathrm{C}}\left(\sigma_{\|, C} \mathcal{J}_{\mathrm{CFC}}\left(\omega_{\mathrm{C}}\right)+\sigma_{\perp, C} \mathcal{J}_{\mathrm{CF} \sigma_{\perp}}\left(\omega_{\mathrm{C}}\right)\right) \\
\eta_{F}= & \mathcal{R}\left(\hat{\mathrm{F}}_{\mathrm{x}} \leftrightarrow 2 \hat{\mathrm{F}}_{\mathrm{x}} \hat{\mathrm{C}}_{\mathrm{z}}\right)=\mathcal{R}\left(\hat{\mathrm{F}}_{\mathrm{y}} \leftrightarrow 2 \hat{\mathrm{F}}_{\mathrm{y}} \hat{\mathrm{C}}_{\mathrm{z}}\right)=\mathcal{R}\left(\hat{\mathrm{F}}^{+} \leftrightarrow 2 \hat{\mathrm{F}}^{+} \hat{\mathrm{C}}_{\mathrm{z}}\right) \\
= & \frac{d_{\mathrm{CF}}}{6} \omega_{\mathrm{F}}\left(\sigma_{\|, F}\left(4 \mathcal{J}_{\mathrm{CFF}}(0)+3 \mathcal{J}_{\mathrm{CFF}_{\|}}\left(\omega_{\mathrm{F}}\right)\right)+\sigma_{\perp, F}\left(4 \mathcal{J}_{\mathrm{CF} \sigma_{\perp}}(0)+3 \mathcal{J}_{\mathrm{CF} \sigma_{\perp}}\left(\omega_{\mathrm{F}}\right)\right)\right) \\
\eta_{C}= & \mathcal{R}\left(\hat{\mathrm{C}}_{\mathrm{x}} \leftrightarrow 2 \hat{\mathrm{F}}_{\mathrm{z}} \hat{\mathrm{C}}_{\mathrm{x}}\right)=\mathcal{R}\left(\hat{\mathrm{C}}_{\mathrm{y}} \leftrightarrow 2 \hat{\mathrm{F}}_{\mathrm{z}} \hat{\mathrm{C}}_{\mathrm{y}}\right) \\
= & \frac{d_{\mathrm{CF}}}{6} \omega_{\mathrm{C}}\left(\sigma_{\|, C}\left(4 \mathcal{J}_{\mathrm{CFC}}(0)+4 \mathcal{J}_{\mathrm{CFC}}\left(\omega_{\mathrm{C}}\right)\right)+\sigma_{\perp, C}\left(4 \mathcal{J}_{\mathrm{CF} \sigma_{\perp}}(0)+3 \mathcal{J}_{\mathrm{CF} \sigma_{\perp}}\left(\omega_{\mathrm{C}}\right)\right)\right) \\
\mu_{m q}= & \mathcal{R}\left(2 \hat{\mathrm{F}}_{\mathrm{x}} \hat{\mathrm{C}}_{\mathrm{x}} \leftrightarrow 2 \hat{\mathrm{F}}_{\mathrm{y}} \hat{\mathrm{C}}_{\mathrm{y}}\right)=\mathcal{R}\left(2 \hat{\mathrm{F}}_{\mathrm{x}} \hat{\mathrm{C}}_{\mathrm{y}} \leftrightarrow 2 \hat{\mathrm{F}}_{\mathrm{y}} \hat{\mathrm{C}}_{\mathrm{x}}\right) \\
= & \frac{d_{\mathrm{CF}}^{2}}{8}\left(6 \mathcal{J}_{\mathrm{CF}}\left(\omega_{\mathrm{F}}+\omega_{\mathrm{C}}\right)-\mathcal{J}_{\mathrm{CF}}\left(\omega_{\mathrm{F}}-\omega_{\mathrm{C}}\right)\right) \\
& +\frac{4}{9} \omega_{\mathrm{F}} \omega_{\mathrm{C}}\left(\sigma_{\|, F} \sigma_{\|, C} \mathcal{J}_{\sigma_{\|}^{F}, \sigma_{\|}^{C}}(0)+\sigma_{\|, F} \sigma_{\perp, C} \mathcal{J}_{\sigma_{\|}^{F}, \sigma_{\perp}^{C}}(0)+\sigma_{\perp, F} \sigma_{\|, C} \mathcal{J}_{\sigma_{\perp}^{F}, \sigma_{\|}^{C}}(0)+\sigma_{\perp, F} \sigma_{\perp, C} \mathcal{J}_{\sigma_{\perp}^{F}, \sigma_{\perp}^{C}}(0)\right)
\end{aligned}
$$




\subsection{Simulation, processing and analysis of a spectra}

\subsubsection{Theory}

Pulse sequences were simulated with Python. The free-evolution Liouvillian for a ${ }^{13} \mathrm{C}-{ }^{19} \mathrm{~F}$ group is:

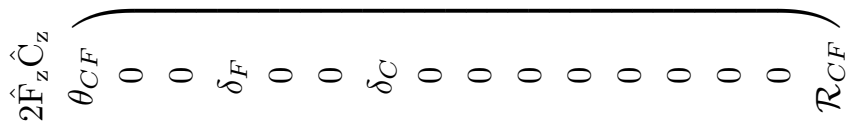

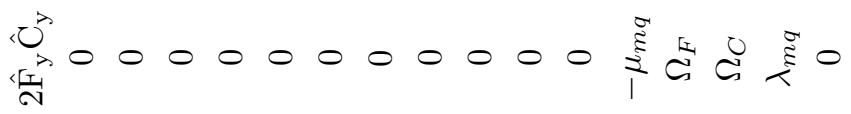

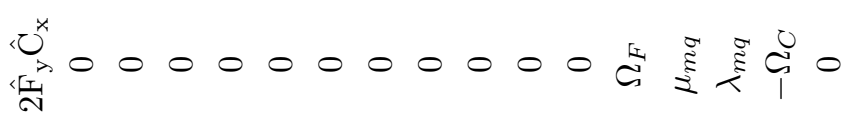

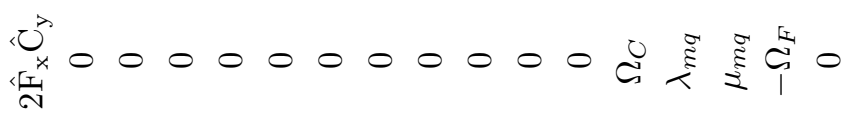

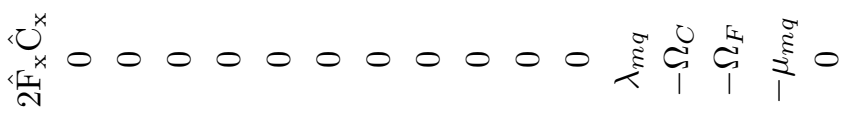

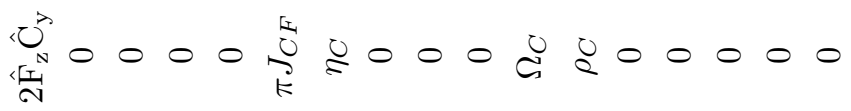

$$
\begin{aligned}
& \underbrace{N}_{N} \\
& \underbrace{N}_{\text {N }} \\
& U_{\text {N }}^{N} \\
& \text { U } 000000 \frac{\tilde{U}^{N}}{N} 00000000 .
\end{aligned}
$$

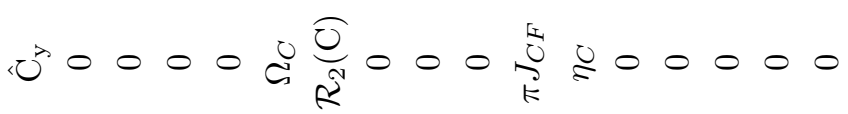

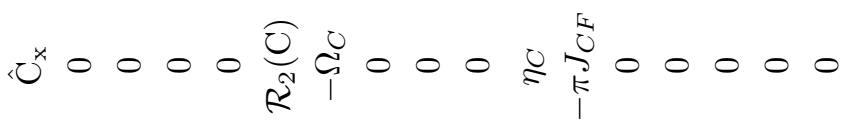

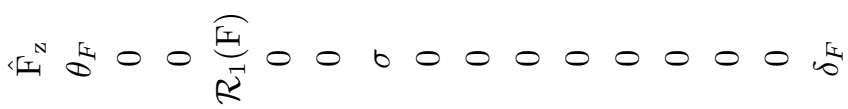

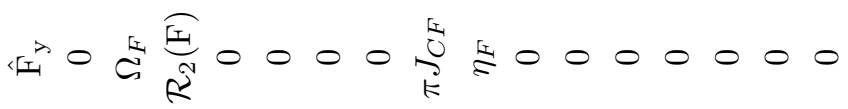

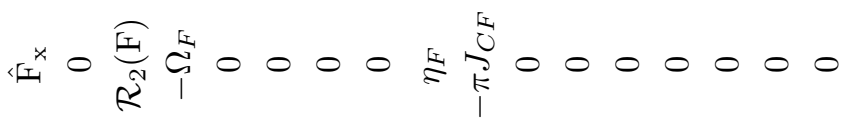

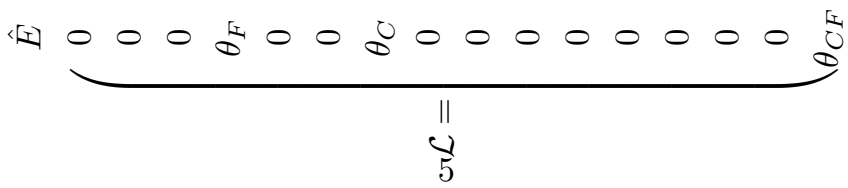


where $J_{C F}=-240 \mathrm{~Hz}$ is the scalar coupling constant between the ${ }^{13} \mathrm{C}$ and ${ }^{19} \mathrm{~F}$ nuclei [2], $\Omega_{i}$ is the offset for nucleus $i$ and:

$$
\begin{aligned}
\theta_{F} & =-2 \times 10^{-10}\left(\omega_{F} \mathcal{R}_{1}(\mathrm{~F})+\omega_{C} \sigma\right), \\
\theta_{C} & =-2 \times 10^{-10}\left(\omega_{C} \mathcal{R}_{1}(\mathrm{C})+\omega_{F} \sigma\right), \\
\theta_{C F} & =-2 \times 10^{-10}\left(\omega_{C} \delta_{C}+\omega_{F} \delta_{F}\right),
\end{aligned}
$$

account for thermal equilibrium. Thus, the effect of the magnetic field on the available polarization is included in the Liouvillian. The factor $10^{-10}$ is used to avoid calculations overflow.

The propagator during a free evolution period is calculated as:

$$
\mathcal{P}_{\text {free }}(\tau)=e^{-\tau \mathcal{L}},
$$

with $\tau$ being the evolution delay. The propagator during an rf pulse is:

$$
\mathcal{P}_{\text {rot }}\left(\tau_{\text {pulse }}\right)=e^{-\tau_{\text {pulse }}\left(\mathcal{L}+\mathcal{L}_{\text {pulse }}\right)},
$$

with $\tau_{\text {pulse }}$ being the pulse length and the Liouvillian for the pulse is:

$\mathcal{L}_{\text {pulse }}=$

$\left(\begin{array}{cccccccccccccccc}0 & 0 & 0 & 0 & 0 & 0 & 0 & 0 & 0 & 0 & 0 & 0 & 0 & 0 & 0 & 0 \\ 0 & 0 & 0 & -\omega_{y}^{F} & 0 & 0 & 0 & 0 & 0 & 0 & 0 & 0 & 0 & 0 & 0 & 0 \\ 0 & 0 & 0 & \omega_{x}^{F} & 0 & 0 & 0 & 0 & 0 & 0 & 0 & 0 & 0 & 0 & 0 & 0 \\ 0 & \omega_{y}^{F} & -\omega_{x}^{F} & & 0 & 0 & 0 & 0 & 0 & 0 & 0 & 0 & 0 & 0 & 0 & 0 \\ 0 & 0 & 0 & 0 & 0 & 0 & -\omega_{y}^{C} & 0 & 0 & 0 & 0 & 0 & 0 & 0 & 0 & 0 \\ 0 & 0 & 0 & 0 & 0 & 0 & \omega_{x}^{C} & 0 & 0 & 0 & 0 & 0 & 0 & 0 & 0 & 0 \\ 0 & 0 & 0 & 0 & \omega_{y}^{C} & -\omega_{x}^{C} & 0 & 0 & 0 & 0 & 0 & 0 & 0 & 0 & 0 & 0 \\ 0 & 0 & 0 & 0 & 0 & 0 & 0 & 0 & 0 & 0 & 0 & \omega_{y}^{C} & -\omega_{x}^{C} & 0 & 0 & -\omega_{y}^{F} \\ 0 & 0 & 0 & 0 & 0 & 0 & 0 & 0 & 0 & 0 & 0 & 0 & 0 & \omega_{y}^{C} & -\omega_{x}^{C} & \omega_{x}^{F} \\ 0 & 0 & 0 & 0 & 0 & 0 & 0 & 0 & 0 & 0 & 0 & \omega_{y}^{F} & 0 & -\omega_{x}^{F} & 0 & -\omega_{y}^{C} \\ 0 & 0 & 0 & 0 & 0 & 0 & 0 & 0 & 0 & 0 & 0 & 0 & \omega_{y}^{F} & 0 & -\omega_{x}^{F} & \omega_{x}^{C} \\ 0 & 0 & 0 & 0 & 0 & 0 & 0 & -\omega_{y}^{C} & 0 & -\omega_{y}^{F} & 0 & 0 & 0 & 0 & 0 & 0 \\ 0 & 0 & 0 & 0 & 0 & 0 & 0 & \omega_{x}^{C} & 0 & 0 & 0-\omega_{y}^{F} & 0 & 0 & 0 & 0 & 0 \\ 0 & 0 & 0 & 0 & 0 & 0 & 0 & 0 & -\omega_{y}^{C} & \omega_{x}^{F} & 0 & 0 & 0 & 0 & 0 & 0 \\ 0 & 0 & 0 & 0 & 0 & 0 & 0 & 0 & \omega_{x}^{C} & 0 & \omega_{x}^{F} & 0 & 0 & 0 & 0 & 0 \\ 0 & 0 & 0 & 0 & 0 & 0 & 0 & \omega_{y}^{F} & -\omega_{x}^{F} & \omega_{y}^{C} & -\omega_{x}^{C} & 0 & 0 & 0 & 0 & 0\end{array}\right)$,

with:

$$
\begin{aligned}
\omega_{x}^{F} & =\frac{\pi}{\tau_{\pi}^{F}} \cos \left(\frac{\pi}{2} \phi_{F}\right) \quad \omega_{y}^{F}=\frac{\pi}{\tau_{\pi}^{F}} \sin \left(\frac{\pi}{2} \phi_{F}\right), \\
\omega_{x}^{C} & =\frac{\pi}{\tau_{\pi}^{C}} \cos \left(\frac{\pi}{2} \phi_{C}\right) \quad \omega_{y}^{C}=\frac{\pi}{\tau_{\pi}^{C}} \sin \left(\frac{\pi}{2} \phi_{C}\right),
\end{aligned}
$$

with $\tau_{\pi}^{\mathrm{i}}$ being the pulse length for a $180^{\circ}$-pulse on nucleus $i$ with phase $\phi_{\mathrm{i}}$. $\phi_{\mathrm{i}}$ equals $0,1,2$ or 3 for a pulse along $\mathrm{x}, \mathrm{y}$, - $\mathrm{x}$ or $-\mathrm{y}$ respectively. Pulse lengths used in the simulations are reported in Table S3.

The gradients used during the ST2-PT block of the single-field experiment [2] (Fig. S3) were simulated by separating the sample into 10,000 slices over $2 \mathrm{~cm}$, modifying the Liouvillian for free evolution in each slice according to the associated magnetic field, and taking the average signal over the whole sample.

\subsubsection{Acquisition details}

When acquiring a spectrum, different user-defined parameters affect the signal intensity and signal-tonoise ratio (acquisition time, sweep width, number of points in the indirect dimension, etc...). In order 
Table S3: Pulse lengths used in the simulation of the experiments. The value of the low-field pulse-lengths is based on our own two-field system $[5,6]$.

\begin{tabular}{|c|cc|cc|}
\hline & \multicolumn{2}{|c|}{ Two-field TROSY } & \multicolumn{2}{c|}{ Single-field TROSY } \\
& Carbon $90^{\circ}$-pulse & Fluorine $90^{\circ}$-pulse & Carbon $90^{\circ}$-pulse & Fluorine $90^{\circ}$-pulse \\
\hline Low field & $10 \mu \mathrm{s}$ & $6 \mu \mathrm{s}$ & - & - \\
High fields & $12 \mu \mathrm{s}$ & $10 \mu \mathrm{s}$ & $12 \mu \mathrm{s}$ & $10 \mu \mathrm{s}$ \\
\hline
\end{tabular}

to get relevant comparison between the simulated pulse sequences, and the range of magnetic fields evaluated in this study, we considered that:

- the spectral width is constant to $10 \mathrm{ppm}$ in both dimension. The number of points is then automatically calculated based on the maximum acquisition time of the associated dimension. Note that in the case of the indirect fluorine dimension, a very low number of points sometimes leads to a different spectral width.

- a maximum acquisition time for the direct carbon dimension of $t_{2, \max }=3 \times T_{2}\left({ }^{13} \mathrm{C}-\mathrm{TROSY}\right)$ where $T_{2}\left({ }^{13} \mathrm{C}-\mathrm{TROSY}\right)$ is the ${ }^{13} \mathrm{C}$-TROSY relaxation rate.

We also considered the pulse lengths to be the same at different magnetic fields (see Table S3).

\subsubsection{Optimization of the recycling delays and simulation of dummy scans}

We define the recycling delay as the sum of the acquisition time and inter-scan delay (d1). This delay allows spins to return to their equilibrium longitudinal state and determines the available polarization at the beginning of the next scan. Having a long recycling delay maximizes the amount of polarization at the expense of experimental time. We optimized the inter-scan delay at each magnetic field for both experiments when the acquisition time is set to $t_{2, \max }$. For this, we simulated 10 scans, without indirect dimension editing, and compared the value of $\frac{F_{z}\left(d_{1}\right)}{\sqrt{t_{2, \text { max }}+d_{1}}}$ of the $11^{\text {th }}$ scan for different values of interscans delays. We devide by $\sqrt{t_{2, \max }+d_{1}}$ since the signal-to-noise ratio evolves as the square-root of the experimental time.

In all the simulations presented here, the inter-scan delays were the optimized delays, and 10 dummy scans were simulated prior to detection to obtain the steady-state fluorine polarization.

\subsubsection{Extracting peak heights from noise-free spectra}

The comparison of the peak heights from one field to the other was used to optimize the magnetic field to record each experiment (see main text). The height of a Lorentzian-shaped peak is proportional to:

$$
\mathrm{H}=\frac{\mathrm{FID}(t=0)}{R_{2}\left({ }^{19} \mathrm{~F}\right) R_{2}\left({ }^{13} \mathrm{C}-\mathrm{TROSY}\right)},
$$

where $\operatorname{FID}(t=0)$ is the signal at time 0 (i.e. the peak area), $R_{2}\left({ }^{13} \mathrm{C}-\mathrm{TROSY}\right)$ is the carbon-TROSY relaxation rate and $R_{2}\left({ }^{19} \mathrm{~F}\right)$ is either the fluorine TROSY single-transition coherence (for the single-field experiment) or the fluorine transverse (for the two-field experiment) relaxation rate. This relationship assumes perfect sampling in both dimensions, which is a good approximation for the direct dimension, but not necessarily in the indirect dimension.

The Liouvillian definition already takes into account the proportionality of the polarization to the magnetic field. However, it does not consider the proportionality of the detected signal to the magnetic field. In addition, at high magnetic fields, the recycling delay becomes longer, leading to potentially 
longer experimental times and lower peak heights per units of time. In order to include these effects, we multiplied the peak height $\mathrm{H}$ by $\sqrt{\frac{\alpha\left(B_{0, i}\right)}{\beta\left(t_{P S, i}\right)}}$ with $B_{0, i}$ the magnetic field of detection for the experiment $i, t_{P S, i}$ the time for one scan of the experiment $i$ without indirect dimension editing and:

$$
\begin{aligned}
& \alpha(B)=\frac{B}{B_{0, \text { ref }}}, \\
& \beta(t)=\frac{t}{t_{P S, \text { ref }}},
\end{aligned}
$$

with $B_{0, \text { ref }}$ and $t_{P S, \text { ref }}$ the magnetic field and experimental time for one scan of the reference experiment without indirect dimension editing. We consider as reference experiment the single-field pulse sequence recorded at $14.1 \mathrm{~T}$ on $3 \mathrm{~F}$-Tyr with a global tumbling correlation time $\tau_{c}=25 \mathrm{~ns}$ (in these conditions, $\left.t_{P S, \text { ref }}=1.52 \mathrm{sec}\right)$. This scaling factor does not consider the effect of accumulating different number of points in the indirect dimension in order to reach a fixed spectral width at different fields. An accurate estimate of the noise level is performed by simulating spectra with noise.

\subsubsection{Processing of the spectra}

The simulated FIDs were processed using the nmrglue Python library [3]. All processing parameters were the same for all spectra (except phases which were adapted from one pulse sequence to the other):

- zero-filling to double the number of points in both dimensions,

- apodization function using the shifted sine-bell function: $\operatorname{sp}(n)=\sin ^{2} \frac{\pi / 2+0.48 \pi n}{N-1}$ with $n$ the data index and $\mathrm{N}$ the number of points in the associated dimension.

\subsection{Noise simulation and signal-to-noise assessment}

When spectra with noise were simulated, a Gaussian-shaped noise centered on 0 was generated using the random.normal function from the Python numpy library and was added to the FID before processing. In each simulation, the same width for the Gaussian distribution was given as input to the random.normal function. In order to consider the effect of the magnetic field on the level of noise, the noise-free FID and the noise were scaled with $\alpha\left(B_{0, i}\right)$ and $\sqrt{\alpha\left(B_{0, i}\right)}$ respectively. This accounts for the proportionality of the noise to $\sqrt{B_{0, i}}$, and the proportionality of the detected signal to $B_{0, i}^{2}$ (note that the effect of the magnetic field on the polarization is already accounted for in the Liouvillian with the thermal correction). In order to take into account the effect of the experimental time on the level of noise, the noise-free FID and the noise were also scaled with $t_{\text {exp }, i} / t_{\text {exp }, \text { ref }}$ and $\sqrt{t_{\text {exp }, i} / t_{\text {exp }, \text { ref }}}$ respectively, with $t_{\text {exp }, i}\left(t_{\text {exp,ref }}\right)$ the total experimental time for the experiment $i$ (for the reference experiment). Here, the reference experiment is the $1 \mathrm{~F}$-TROSY pulse sequence used to record spectra of $3 \mathrm{~F}-\mathrm{Tyr}$ at $14.1 \mathrm{~T}$ with global tumbling correlation time $\tau_{c}=25 \mathrm{~ns}$ and an indirect dimension aquisition time $t_{1, \max }=1.25 \times T_{2}\left({ }^{19} \mathrm{~F}-\mathrm{TROSY}\right)$ with $T_{2}\left({ }^{19} \mathrm{~F}-\right.$ TROSY) the relaxation time for the fluorine TROSY component (experimental time of 4.9 hours).

Evaluation of the signal-to-noise ratio was done using two spectra:

- the spectrum corresponding to the sum of the FID and the noise. This spectrum was used to extract the peak height $I$;

- the spectrum of the noise only. This spectrum was generated by processing the random noise using the same parameters as for the complete FID. This spectrum was used to extract the standard deviation of the noise $\sigma_{\text {noise }}$.

The signal-to-noise ratio (SNR) is then given by:

$$
\mathrm{SNR}=\frac{I}{\sigma_{\text {noise }}} .
$$


This computational procedure ensures that no parts of the peak are considered in the estimation of the noise (which is particularly critical when the TROSY selection is not optimal) and that sufficient points are included to obtain an accurate estimate of $\sigma_{\text {noise }}$ (a critical point when a low number of points is used in the indirect dimension).

The level of noise was choosen such that it would visually reproduce the noise in spectra already published [2]. We simulated the single-field experiment using the same experimental parameters as the ones used to record the spectrum of the maltose binding protein (MBP) until the noise was visually similar to the one reported in the original paper (signal-to-noise ratio of 25, Fig. S11 and [2]). Since the MBP spectrum was recorded with 80 scans [2], the spectra and noise level were scaled with 80 and $\sqrt{80}$ respectively. Thus, the scaling of the FID and noise level by $80 \times t_{\text {exp }, i} / t_{\text {exp }, \text { ref }}$ and $\sqrt{80 \times t_{\text {exp }, i} / t_{\text {exp }, \text { ref }}}$ respectively leads to spectra with a number of scans leading to the same experimental time for each of them. 


\section{Product operator analysis of the pulse sequences}

\subsection{Single-field TROSY experiment}

In the following, relaxation effects are neglected. The pulse sequence [2] is shown in Figure S3. The first carbon pulse and gradient $g_{1}$ remove carbon polarization. After the pulse $\varphi_{1}$ :

$$
\begin{aligned}
\hat{\mathrm{F}}_{\mathrm{x}} \stackrel{\Omega_{F} t_{1} \hat{F}_{z}+2 \pi J_{C F} t_{1} \hat{F}_{z} \hat{C}_{z}}{\longrightarrow} & \hat{\mathrm{F}}_{\mathrm{x}} \cos \left(\Omega_{F} t_{1}\right) \cos \left(\pi J_{C F} t_{1}\right)+\hat{\mathrm{F}}_{\mathrm{y}} \sin \left(\Omega_{F} t_{1}\right) \cos \left(\pi J_{C F} t_{1}\right) \\
& +2 \hat{\mathrm{F}}_{\mathrm{y}} \hat{\mathrm{C}}_{\mathrm{z}} \cos \left(\Omega_{F} t_{1}\right) \sin \left(\pi J_{C F} t_{1}\right)-2 \hat{\mathrm{F}}_{\mathrm{x}} \hat{\mathrm{C}}_{\mathrm{z}} \sin \left(\Omega_{F} t_{1}\right) \sin \left(\pi J_{C F} t_{1}\right),
\end{aligned}
$$

where $\Omega_{F}$ is the offset frequency for the fluorine nucleus and $J_{C F}$ the scalar coupling constant between the carbon and fluorine. The Single Transition-to-Single Transition Polarization Transfer (ST2-PT) block [4] converts these operators into observable carbon magnetization (recall that the coupling constant is negative):

$$
\begin{aligned}
& \hat{\mathrm{F}}_{\mathrm{x}} \stackrel{\frac{\pi}{2} \hat{C}_{y}}{\longrightarrow} \hat{\mathrm{F}}_{\mathrm{x}} \stackrel{\pi\left(\hat{F}_{x}+\hat{C}_{x}\right)-\pi \hat{F}_{z} \hat{C}_{z}}{\longrightarrow}-2 \hat{\mathrm{F}}_{\mathrm{y}} \hat{\mathrm{C}}_{\mathrm{z}} \stackrel{\frac{\pi}{2}\left(\hat{C}_{x}-\hat{F}_{y}\right)}{\longrightarrow} 2 \hat{\mathrm{F}}_{\mathrm{y}} \hat{\mathrm{C}}_{\mathrm{y}} \stackrel{\pi\left(\hat{F}_{x}+\hat{C}_{x}\right)-\pi \hat{F}_{z} \hat{C}_{z}}{\longrightarrow} 2 \hat{\mathrm{F}}_{\mathrm{y}} \hat{\mathrm{C}}_{\mathrm{y}} \stackrel{\frac{\pi}{2}}{\stackrel{F_{x}}{\longrightarrow}} 2 \hat{\mathrm{F}}_{\mathrm{z}} \hat{\mathrm{C}}_{\mathrm{y}}, \\
& \hat{\mathrm{F}}_{\mathrm{y}} \stackrel{\frac{\pi}{2} \hat{C}_{y}}{\longrightarrow} \hat{\mathrm{F}}_{\mathrm{y}} \stackrel{\pi\left(\hat{F}_{x}+\hat{C}_{x}\right)-\pi \hat{F}_{z} \hat{C}_{z}}{\longrightarrow}-2 \hat{\mathrm{F}}_{\mathrm{x}} \hat{\mathrm{C}}_{\mathrm{z}} \stackrel{\frac{\pi}{2}\left(\hat{C}_{x}-\hat{F}_{y}\right)}{\longrightarrow} 2 \hat{\mathrm{F}}_{\mathrm{z}} \hat{\mathrm{C}}_{\mathrm{y}} \stackrel{\pi\left(\hat{F}_{x}+\hat{C}_{x}\right)-\pi \hat{F}_{z} \hat{C}_{z}}{\longrightarrow} \hat{\mathrm{C}}_{\mathrm{x}} \stackrel{\frac{\pi}{2} \hat{F}_{x}}{\longrightarrow} \hat{\mathrm{C}}_{\mathrm{x}}, \\
& 2 \hat{\mathrm{F}}_{\mathrm{x}} \hat{\mathrm{C}}_{\mathrm{z}} \stackrel{\frac{\pi}{2} \hat{C}_{y}}{\longrightarrow} 2 \hat{\mathrm{F}}_{\mathrm{x}} \hat{\mathrm{C}}_{\mathrm{x}} \stackrel{\pi\left(\hat{F}_{x}+\hat{C}_{x}\right)-\pi \hat{F}_{z} \hat{C}_{z}}{\longrightarrow} 2 \hat{\mathrm{F}}_{\mathrm{x}} \hat{\mathrm{C}}_{\mathrm{x}} \stackrel{\frac{\pi}{2}\left(\hat{C}_{x}-\hat{F}_{y}\right)}{\longrightarrow} 2 \hat{\mathrm{F}}_{\mathrm{z}} \hat{\mathrm{C}}_{\mathrm{x}} \stackrel{\pi\left(\hat{F}_{x}+\hat{C}_{x}\right)-\pi \hat{F}_{z} \hat{C}_{z}}{\longrightarrow} \hat{\mathrm{C}}_{\mathrm{y}} \stackrel{\frac{\pi}{2}}{\stackrel{F_{x}}{\longrightarrow}} \hat{\mathrm{C}}_{\mathrm{y}}, \\
& 2 \hat{\mathrm{F}}_{\mathrm{y}} \hat{\mathrm{C}}_{\mathrm{z}} \stackrel{\frac{\pi}{2} \hat{C}_{y}}{\longrightarrow} 2 \hat{\mathrm{F}}_{\mathrm{y}} \hat{\mathrm{C}}_{\mathrm{x}} \stackrel{\pi\left(\hat{F}_{x}+\hat{C}_{x}\right)-\pi \hat{F}_{z} \hat{C}_{z}}{\longrightarrow}-2 \hat{\mathrm{F}}_{\mathrm{y}} \hat{\mathrm{C}}_{\mathrm{x}} \stackrel{\frac{\pi}{2}\left(\hat{C}_{x}-\hat{F}_{y}\right)}{\longrightarrow}-2 \hat{\mathrm{F}}_{\mathrm{y}} \hat{\mathrm{C}}_{\mathrm{x}} \stackrel{\pi\left(\hat{F}_{x}+\hat{C}_{x}\right)-\pi \hat{F}_{z} \hat{C}_{z}}{\longrightarrow} 2 \hat{\mathrm{F}}_{\mathrm{y}} \hat{\mathrm{C}}_{\mathrm{x}} \stackrel{\frac{\pi}{2} \hat{F}_{x}}{\longrightarrow} 2 \hat{\mathrm{F}}_{\mathrm{z}} \hat{\mathrm{C}}_{\mathrm{x}} .
\end{aligned}
$$

During the acquisition time $t_{2}$, the signal has the form:

$$
\mathcal{S}_{y, x}^{E}\left(t_{1}, t_{2}\right) \propto \sin \left(\Omega_{F} t_{1}-\pi J_{C F} t_{2}\right) e^{i\left(\Omega_{C} t_{2}-\pi J_{C F} t_{1}\right)},
$$

where the notation $\mathcal{S}_{y, x}^{E}$ denotes the signal recorded for the set of phases $\varphi_{1}=y$ and $\varphi_{\text {rec }}=x$ during the echo part of the sequence. The second cycle $\left(\varphi_{1}=-y, \varphi_{\text {rec }}=-x\right)$ produces the same FID. The last two cycles $\left(\varphi_{1}, \varphi_{\text {rec }}\right)$ lead to the signal:

$$
\begin{aligned}
& \mathcal{S}_{x,-y}^{E}\left(t_{1}, t_{2}\right)=\mathcal{S}_{-x, y}^{E}\left(t_{1}, t_{2}\right), \\
& \mathcal{S}_{x,-y}^{E}\left(t_{1}, t_{2}\right) \propto-i \cos \left(\Omega_{F} t_{1}-\pi J_{C F} t_{2}\right) e^{i\left(\Omega_{C} t_{2}-\pi J_{C F} t_{1}\right)} .
\end{aligned}
$$

In the echo part of the experiment, this leads to a signal of the form:

$$
\begin{aligned}
\mathcal{S}^{E}\left(t_{1}, t_{2}\right) & =\sum_{\left(\varphi_{1}, \varphi_{\mathrm{rec}}\right)^{E}} \mathcal{S}_{\left(\varphi_{1}, \varphi_{\mathrm{rec}}\right)}^{E}\left(t_{1}, t_{2}\right), \\
& \propto-i e^{i\left(\Omega_{F}-\pi J_{C F}\right) t_{1}} e^{i\left(\Omega_{C}-\pi J_{C F}\right) t_{2}},
\end{aligned}
$$

where the factor $-i$ only introduces a $-90^{\circ}$ phase shift.

During the anti-echo acquisition, the ST2-PT block converts the operators into:

$$
\begin{gathered}
\hat{\mathrm{F}}_{\mathrm{x}} \stackrel{\mathrm{ST} 2 \mathrm{PT}}{\longrightarrow} 2 \hat{\mathrm{F}}_{\mathrm{z}} \hat{\mathrm{C}}_{\mathrm{y}}, \\
\hat{\mathrm{F}}_{\mathrm{y}} \stackrel{\mathrm{ST} 2 \mathrm{PT}}{\longrightarrow}-\hat{\mathrm{C}}_{\mathrm{x}}, \\
2 \hat{\mathrm{F}}_{\mathrm{x}} \hat{\mathrm{C}}_{\mathrm{z}} \stackrel{\mathrm{ST} 2 \mathrm{PT}}{\longrightarrow} \hat{\mathrm{C}}_{\mathrm{y}}, \\
2 \hat{\mathrm{F}}_{\mathrm{y}} \hat{\mathrm{C}}_{\mathrm{z}} \stackrel{\mathrm{ST} 2 \mathrm{PT}}{\longrightarrow}-2 \hat{\mathrm{F}}_{\mathrm{z}} \hat{\mathrm{C}}_{\mathrm{x}},
\end{gathered}
$$

leading to:

$$
\begin{aligned}
& \mathcal{S}_{(-y, x)}^{A E}\left(t_{1}, t_{2}\right) \propto \sin \left(\Omega_{F} t_{1}+\pi J_{C F} t_{2}\right) e^{i\left(\Omega_{C} t_{2}+\pi J_{C F} t_{1}\right)}, \\
& \mathcal{S}_{(x,-y)}^{A E}\left(t_{1}, t_{2}\right) \propto i \cos \left(\Omega_{F} t_{1}+\pi J_{C F} t_{2}\right) e^{i\left(\Omega_{C} t_{2}+\pi J_{C F} t_{1}\right)},
\end{aligned}
$$

where the exponent $A E$ refers to the anti-echo acquisition of the sequence. Finally:

$$
\mathcal{S}^{A E}\left(t_{1}, t_{2}\right) \propto i e^{i\left(\Omega_{F}+\pi J_{C F}\right) t_{1}} e^{i\left(\Omega_{C}-\pi J_{C F}\right) t_{2}} .
$$

The Rance-Kay processing leads to the final spectrum with selection of the ${ }^{19} \mathrm{~F}$-TROSY component in the indirect dimension. 


\subsection{Two-field TROSY experiment}

In the following, relaxation effects are neglected. The pulse sequence is shown in Fig. 2 of the main text. Polarization occurs at high field and the magnetization is stored and shuttled as $\hat{F}_{z}$ operator. Indirect dimension evolution is performed in a semi-constant time fashion such that scalar-coupling evolution is refocussed. The phase cycle on $\varphi_{2}$ allows selection of the cosine or sine evolving part which is further transfered back to the $2 \hat{F}_{z} \hat{C}_{z}$ operator for shuttling to high field. The first carbon pulse creates anti-phase carbon magnetization which further evolves under scalar coupling during a time $\mathrm{T} / 2$ :

$$
2 \hat{\mathrm{F}}_{\mathrm{z}} \hat{\mathrm{C}}_{\mathrm{z}} \stackrel{\pi \hat{F}_{x}}{\longrightarrow}-2 \hat{\mathrm{F}}_{\mathrm{z}} \hat{\mathrm{C}}_{\mathrm{z}} \stackrel{\frac{\pi}{2} \hat{C}_{x}}{\longrightarrow} 2 \hat{\mathrm{F}}_{\mathrm{z}} \hat{\mathrm{C}}_{\mathrm{y}} \stackrel{\pi\left(\hat{F}_{x}+\hat{C}_{x}\right)-\frac{\pi}{2} \hat{F}_{z} \hat{C}_{z}}{\longrightarrow} \frac{\sqrt{2}}{2}\left(2 \hat{\mathrm{F}}_{\mathrm{z}} \hat{\mathrm{C}}_{\mathrm{y}}+\hat{\mathrm{C}}_{\mathrm{x}}\right)
$$

The phase cycle on $\varphi_{3}$ and the use of two fluorine $180^{\circ}$ pulses allow selection of the carbon-TROSY component.

$$
\begin{array}{r}
\varphi_{3}=\frac{\pi}{4}: \frac{\sqrt{2}}{2}\left(2 \hat{\mathrm{F}}_{\mathrm{z}} \hat{\mathrm{C}}_{\mathrm{y}}+\hat{\mathrm{C}}_{\mathrm{x}}\right) \stackrel{\varphi_{3}}{\longrightarrow} \frac{\sqrt{2}}{2}\left[\frac{1}{2}\left(2 \hat{\mathrm{F}}_{\mathrm{z}} \hat{\mathrm{C}}_{\mathrm{x}}+2 \hat{\mathrm{F}}_{\mathrm{z}} \hat{\mathrm{C}}_{\mathrm{y}}\right)+\frac{\sqrt{2}}{2} 2 \hat{\mathrm{F}}_{\mathrm{z}} \hat{\mathrm{C}}_{\mathrm{z}}+\frac{1}{2}\left(\hat{\mathrm{C}}_{\mathrm{x}}+\hat{\mathrm{C}}_{\mathrm{y}}\right)-\frac{\sqrt{2}}{2} \hat{\mathrm{C}}_{\mathrm{z}}\right] \\
\stackrel{\frac{\pi}{2} \hat{\mathrm{C}}_{y}}{\longrightarrow} \frac{\sqrt{2}}{2}\left[-\frac{1}{2}\left(2 \hat{\mathrm{F}}_{\mathrm{z}} \hat{\mathrm{C}}_{\mathrm{z}}-2 \hat{\mathrm{F}}_{\mathrm{z}} \hat{\mathrm{C}}_{\mathrm{y}}\right)+\frac{\sqrt{2}}{2} 2 \hat{\mathrm{F}}_{\mathrm{z}} \hat{\mathrm{C}}_{\mathrm{x}}-\frac{1}{2}\left(\hat{\mathrm{C}}_{\mathrm{z}}-\hat{\mathrm{C}}_{\mathrm{y}}\right)-\frac{\sqrt{2}}{2} \hat{\mathrm{C}}_{\mathrm{x}}\right], \\
\varphi_{3}=\frac{5 \pi}{4}: \frac{\sqrt{2}}{2}\left(2 \hat{\mathrm{F}}_{\mathrm{z}} \hat{\mathrm{C}}_{\mathrm{y}}+\hat{\mathrm{C}}_{\mathrm{x}}\right) \stackrel{\varphi_{3}}{\longrightarrow} \frac{\sqrt{2}}{2}\left[\frac{1}{2}\left(2 \hat{\mathrm{F}}_{\mathrm{z}} \hat{\mathrm{C}}_{\mathrm{x}}+2 \hat{\mathrm{F}}_{\mathrm{z}} \hat{\mathrm{C}}_{\mathrm{y}}\right)-\frac{\sqrt{2}}{2} 2 \hat{\mathrm{F}}_{\mathrm{z}} \hat{\mathrm{C}}_{\mathrm{z}}+\frac{1}{2}\left(\hat{\mathrm{C}}_{\mathrm{x}}+\hat{\mathrm{C}}_{\mathrm{y}}\right)+\frac{\sqrt{2}}{2} \hat{\mathrm{C}}_{\mathrm{z}}\right] \\
\stackrel{\frac{\pi}{2} \hat{C}_{y}}{\longrightarrow} \frac{\sqrt{2}}{2}\left[-\frac{1}{2}\left(2 \hat{\mathrm{F}}_{\mathrm{z}} \hat{\mathrm{C}}_{\mathrm{z}}-2 \hat{\mathrm{F}}_{\mathrm{z}} \hat{\mathrm{C}}_{\mathrm{y}}\right)-\frac{\sqrt{2}}{2} 2 \hat{\mathrm{F}}_{\mathrm{z}} \hat{\mathrm{C}}_{\mathrm{x}}-\frac{1}{2}\left(\hat{\mathrm{C}}_{\mathrm{z}}-\hat{\mathrm{C}}_{\mathrm{y}}\right)+\frac{\sqrt{2}}{2} \hat{\mathrm{C}}_{\mathrm{x}}\right] .
\end{array}
$$

Changing the receiver phase from $-x$ to $+x$ leads to the selection of the $2 \hat{\mathrm{F}}_{\mathrm{z}} \hat{\mathrm{C}}_{\mathrm{x}}-\hat{\mathrm{C}}_{\mathrm{x}}$ operator before detection (i.e. the TROSY component). Reconstruction of the indirect dimension is done after Fourier transform of the direct dimension as:

$$
\mathcal{S}\left(t_{1}, \omega_{2}\right)=\mathcal{S}_{\varphi_{1}=x}\left(t_{1}, \omega_{2}\right)+i \mathcal{S}_{\varphi_{1}=y}\left(t_{1}, \omega_{2}\right)
$$

with $\mathcal{S}_{\varphi_{1}=k}\left(t_{1}, \omega_{2}\right)$ being the Fourier-transformed signal in the direct dimension recorded with phase $\varphi_{1}=k$. 


\section{Additional Figures}
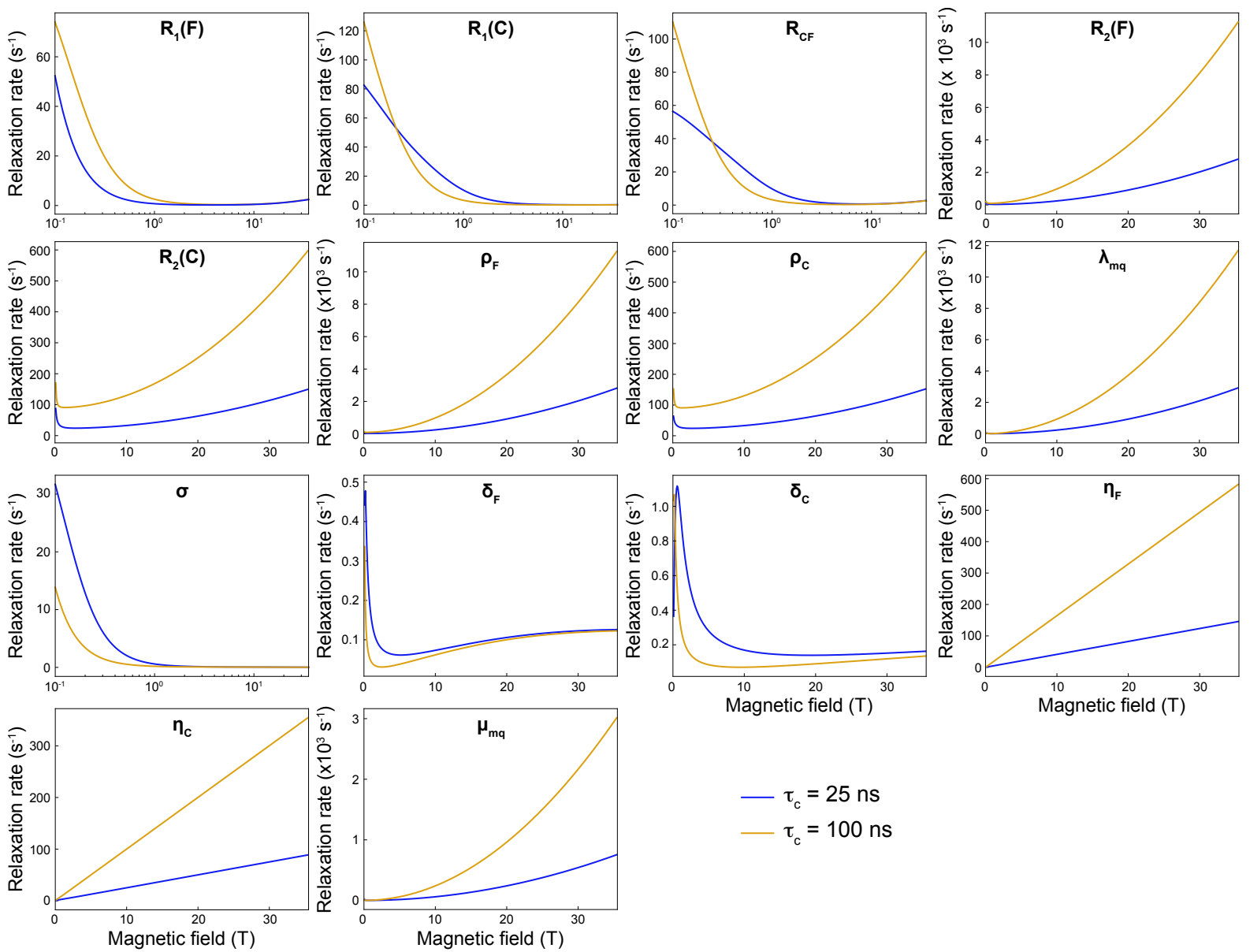

$$
\begin{aligned}
-\tau_{c} & =25 \mathrm{~ns} \\
-\tau_{c} & =100 \mathrm{~ns}
\end{aligned}
$$

Figure S1: Magnetic field variations of the auto- and cross-relaxation rates of the fluorine and carbon operators forming the Liouville space for different values of correlation time $\tau_{c}$ and for an isolated ${ }^{13} \mathrm{C}-{ }^{19} \mathrm{~F}$ spin pair. Calculations were performed using the 3-fluorotyrosine CSA tensor parameters. 


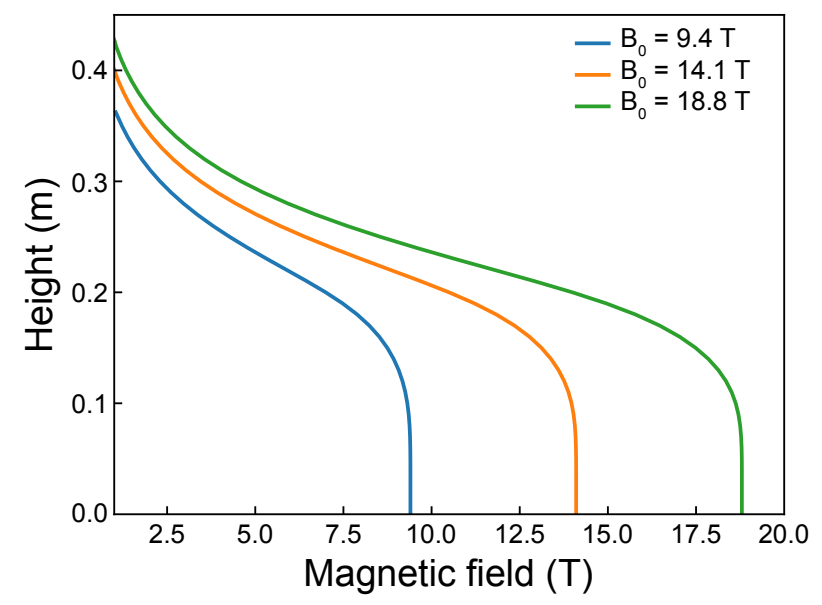

Figure S2: Examples of magnetic field profiles used in the simulations. The field profile for a spectrometer operating at $14.1 \mathrm{~T}$ (orange) is taken as a reference and matches our own $600 \mathrm{MHz}$ two-field NMR spectrometer. The magnetic field profiles for spectrometers operating at $9.4 \mathrm{~T}$ (blue) and $18.8 \mathrm{~T}$ (green) are calculated by multiplying the reference profile (orange) by a factor $\alpha\left(B_{0}\right)=B_{0} / 14.1$ where $B_{0}$ is the operating magnetic field.

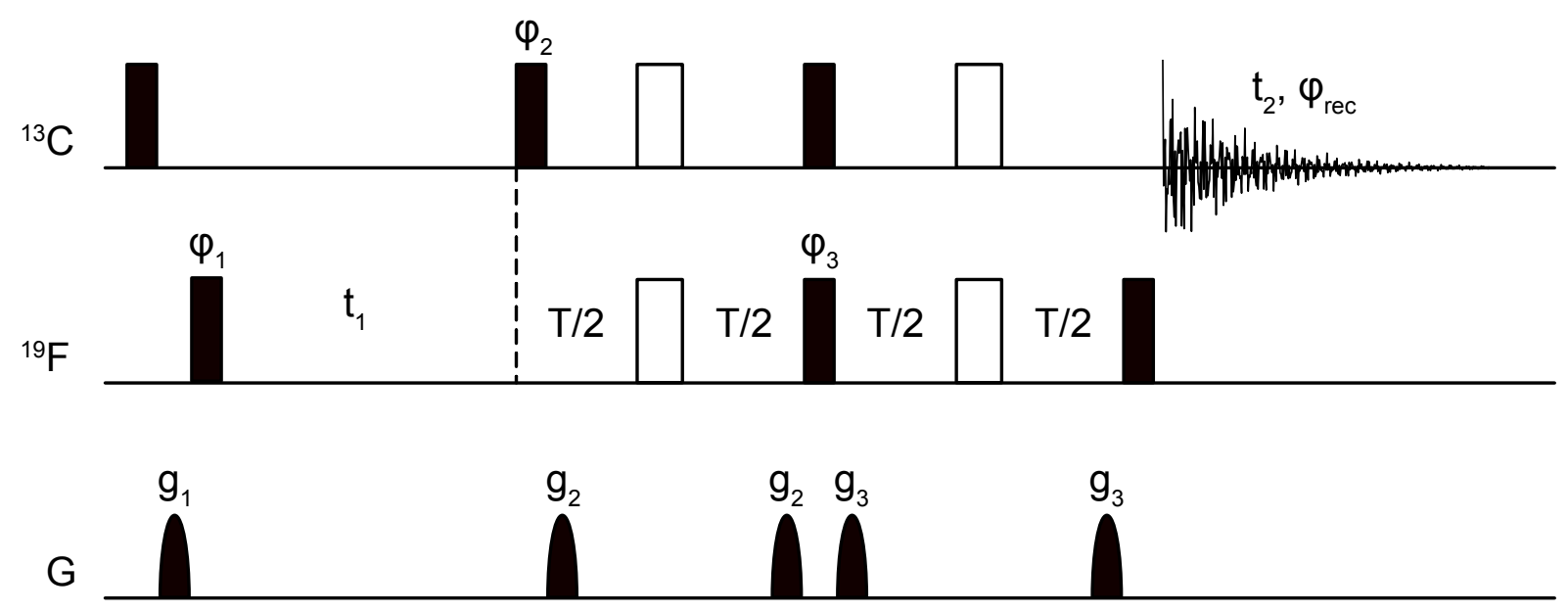

Figure S3: Single-field TROSY pulse-sequence for ${ }^{13} \mathrm{C}-{ }^{19} \mathrm{~F}$ aromatic groups. This sequence can be modified to include proton decoupling during the $t_{1}$ and $t_{2}$ evolution period as detailed in the original publication [2]. Black narrow (resp. wide white) rectangles represent $90^{\circ}$ (resp. 180 ${ }^{\circ}$ ) pulses. Phases are aligned along $\mathrm{x}$ if not otherwise stated. Phase cycles are as follows: $\varphi_{1}=(\mathrm{y},-\mathrm{y}, \mathrm{x},-\mathrm{x})$, and $\varphi_{\text {rec }}=(\mathrm{x},-$ $\mathrm{x},-\mathrm{y}, \mathrm{y})$. Pulses $\varphi_{2}$ and $\varphi_{3}$ select the TROSY component. In the case of the 3 -fluorotyrosine, $\varphi_{2}=\mathrm{y}$ and $\varphi_{3}=-\mathrm{y}$, while $\varphi_{2}=-\mathrm{y}$ and $\varphi_{3}=-\mathrm{y}$ for the 4 -fluorophenylalanine. The indirect dimension is recorded in an Echo/Anti-Echo scheme by recording another set of experiment with following phases: $\varphi_{1}=(-\mathrm{y}, \mathrm{y}, \mathrm{x},-$ x), $\varphi_{2}=$-y (3-fluorotyrosine) or $\varphi_{2}=\mathrm{y}$ (4-fluorophenylalanine), $\varphi_{3}=\mathrm{y}$ and the same receiver phase. The duration of the delay $\mathrm{T}=1 /(2 \mathrm{~J})$. Gradient $\mathrm{g}_{1}$ is a cleaning gradient and was simulated by setting the initial Liouvillian to Fz population only. Length of the gradients is $750 \mu$ s with values $24.75 \mathrm{G}^{-\mathrm{cm}^{-1}}$ for $\mathrm{g}_{2}$ and $27.5 \mathrm{G} . \mathrm{cm}^{-1}$ for $\mathrm{g}_{3}$. 


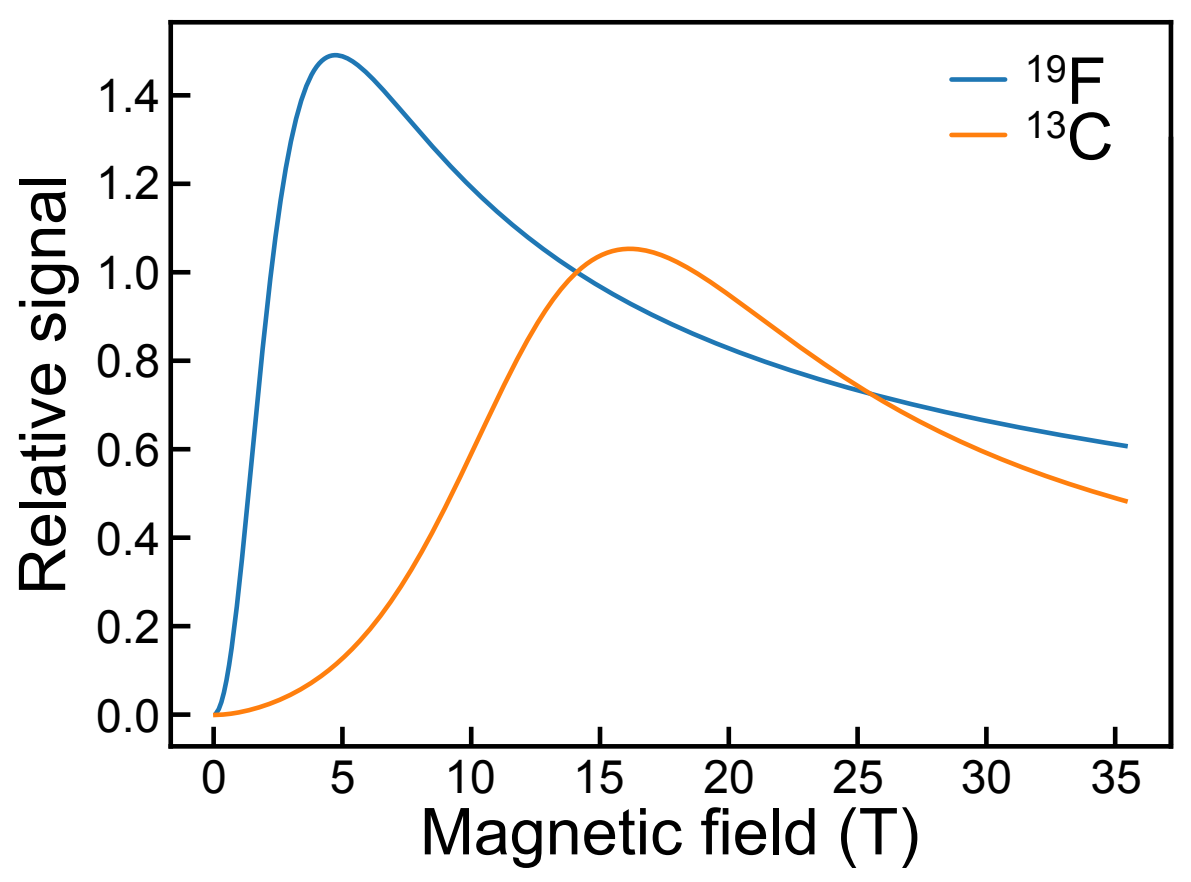

Figure S4: Field optimization following the approach of Ref. [7]. The relative signal for a nucleus $X$ at a magnetic field $B_{0}$ is given by $B_{0}^{3 / 2} \times T_{2}(X)$ where $T_{2}(X)$ is the transverse relaxation time of $X$ and scaled so that the relative signal is 1.0 at $14.1 \mathrm{~T}$. These calculations have been performed for a global tumbling correlation time of $25 \mathrm{~ns}$ and the CSA tensor parameters of 3F-Tyr.
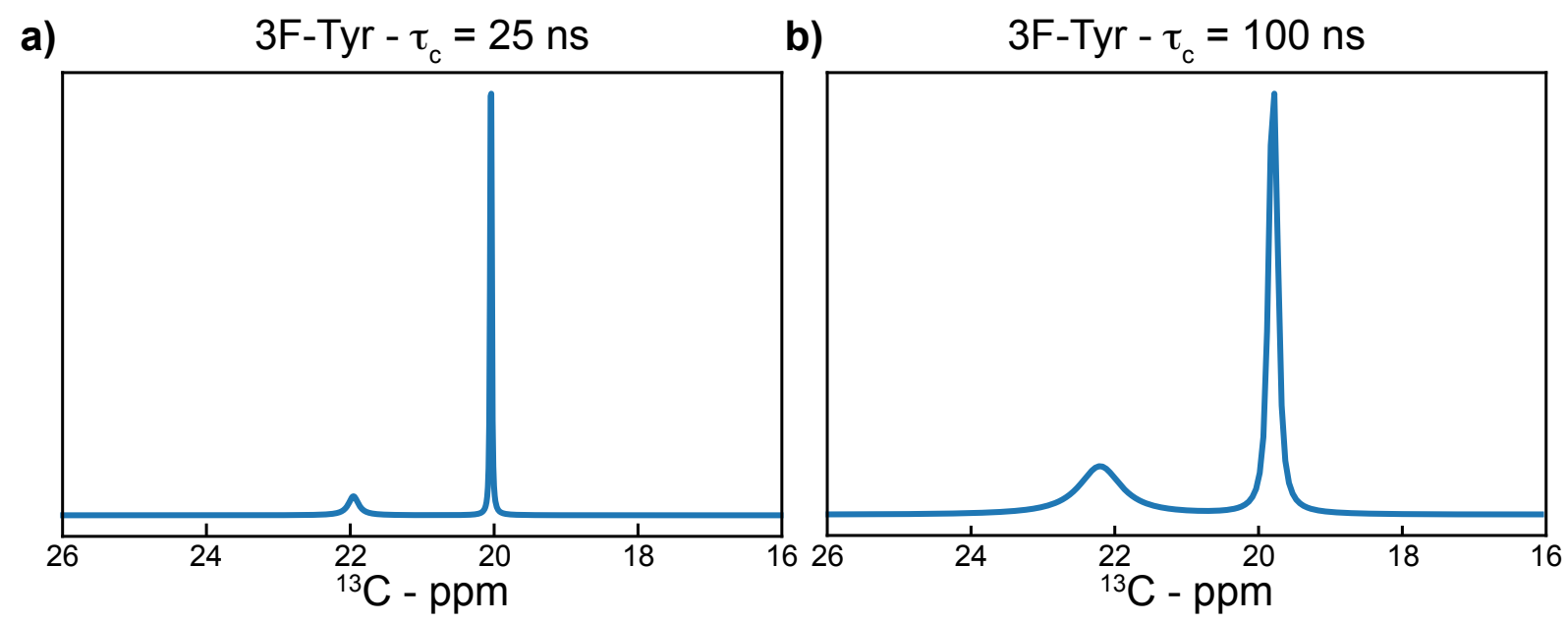

Figure S5: The 1F-TROSY experiment does not perfectly selects the carbon-TROSY peak. Carbon cross-section of two-dimensional spectra simulated with the 1F-TROSY pulse sequence for 3F-Tyr with a global tumbling correlation time $\tau_{c}=25 \mathrm{~ns}(\mathbf{a})$ and $\tau_{c}=100 \mathrm{~ns}(\mathbf{b})$. 


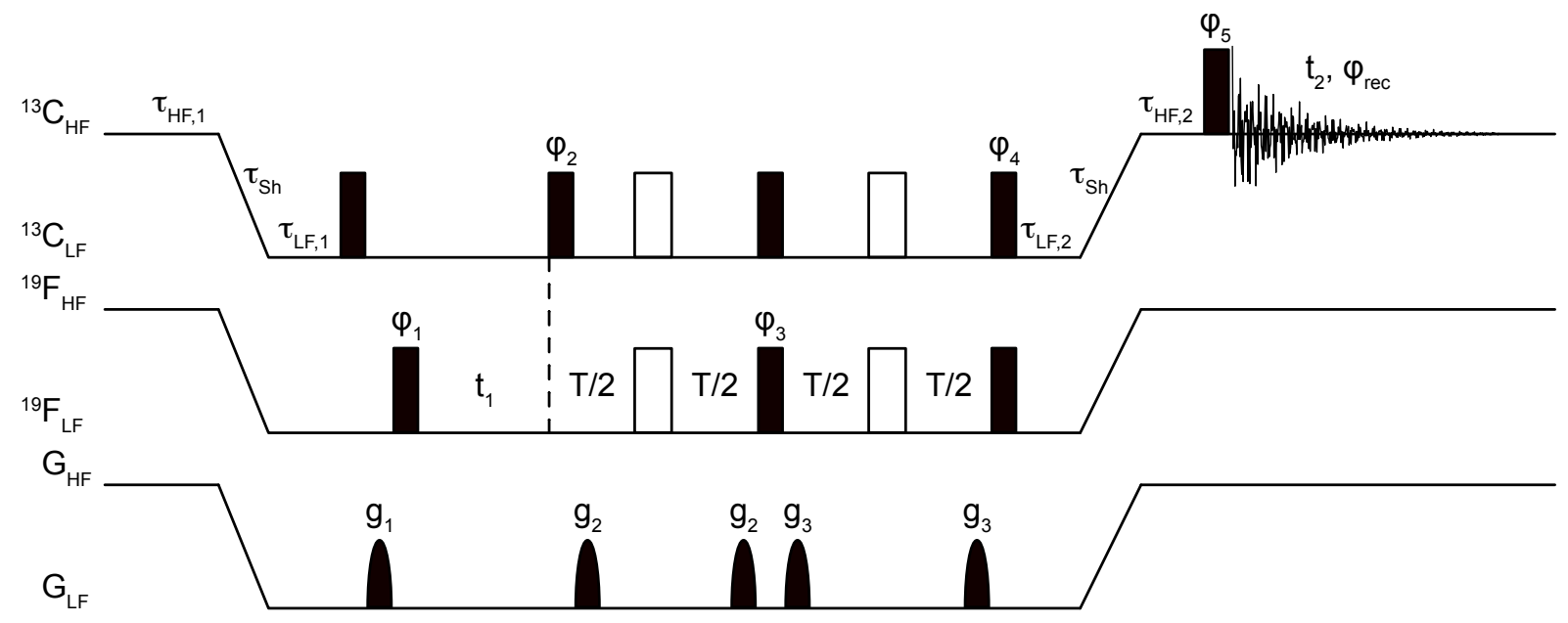

Figure S6: Two-field TROSY pulse selection with selection of both ${ }^{19} \mathrm{~F}$ and ${ }^{13} \mathrm{C}$-TROSY components in ${ }^{13} \mathrm{C}-{ }^{19} \mathrm{~F}$ aromatic groups. Black narrow (respectively wide white) rectangles represent $90^{\circ}$ (respectively $180^{\circ}$ ) pulses. Phases are aligned along the $\mathrm{x}$-axis of the rotating frame unless otherwise stated. Phase cycles are as follows: $\varphi_{1}=(y,-y, x,-x, y,-y, x,-x), \varphi_{4}=(x, x, x, x, y, y, y, y)$, $\varphi_{5}=(-x,-x,-x,-x,-y,-y,-y,-y)$ and $\varphi_{\text {rec }}=(x,-x,-y, y, x,-x,-y, y)$. In order to select for the TROSY peak, phases $\varphi_{2}$ and $\varphi_{3}$ have to be adjusted to either $y$ and $-y$ for selection of $\hat{F}^{+}-2 \hat{F}^{+} \hat{C}_{z}$ (3F-Tyr) or to $-y$ and $-y$ for selection of $\hat{F}^{+}+2 \hat{F}^{+} \hat{C}_{z}$ (4F-Phe). Frequency discrimination in the indirect dimension is performed using the Echo/Anti-Echo acquisition scheme, with phases $\varphi_{1}=(-y, y, x,-x,-y, y, x,-x)$ and the phases $\varphi_{2}$ and $\varphi_{3}$ shifted by $180^{\circ}$. Length of the gradient $g_{2}$

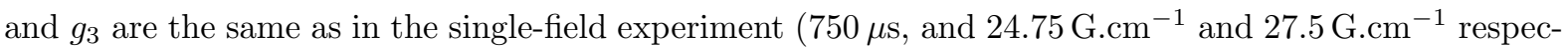
tively). This pulse sequence can be modified to include proton decoupling during fluorine and carbon chemical shift evolution periods. The horizontal line breaks represent the shuttling periods from one field to the other. Time $\tau_{S h}$ is the shuttling delay, $\tau_{H F, 1}$ and $\tau_{L F, 2}$ are waiting delays before shuttling and $\tau_{H F, 2}$ and $\tau_{L F, 1}$ are waiting delays after shuttling, as detailed in the main text. 

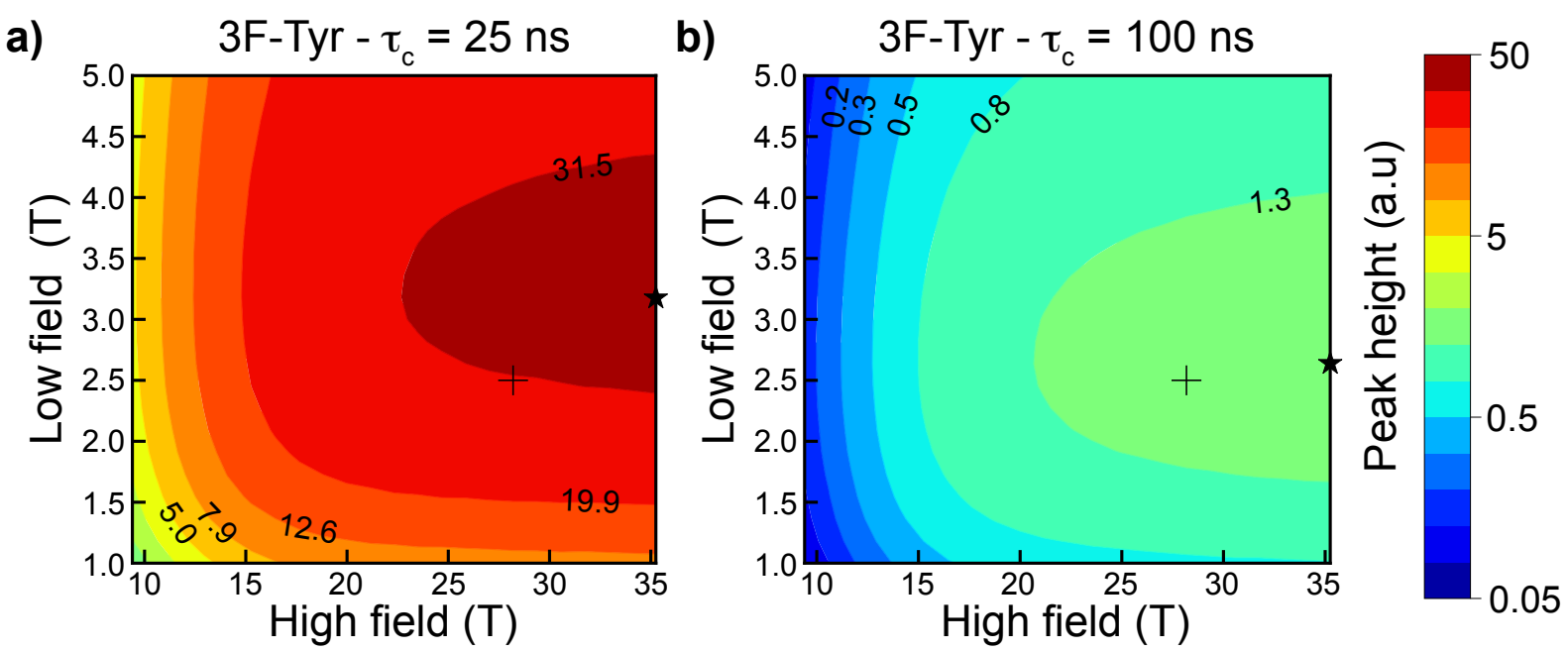

Figure S7: Field optimization of the 2F-ST2PT experiment. Expected peak height for the 3F-Tyr for a global tumbling correlation time $\tau_{c}$ of $25 \mathrm{~ns}(\mathbf{a})$ and $100 \mathrm{~ns}(\mathbf{b})$. The highest peak-height position is indicated with a star. The optimal low-field remains the same as for the two-field experiment discussed in the main text $(2.5 \mathrm{~T})$. Interestingly, the optimial high field is higher. This is due to an increase in the ${ }^{19} \mathrm{~F}$ longitudinal relaxation rate (Fig. S1) leading to a smaller recycling delay and hence lower experimental time. We simulated the experiment at the highest commercially available magnetic field at the time of writing (i.e. $28.2 \mathrm{~T}$ ).
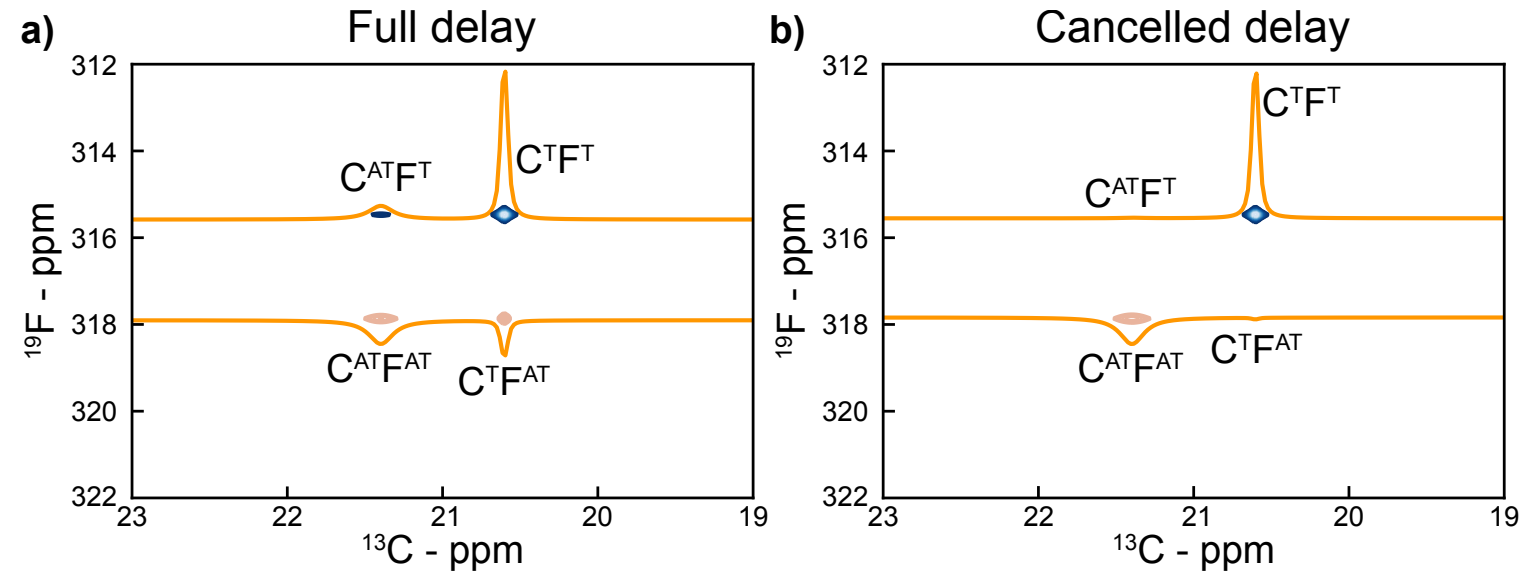

Figure S8: The 2F-ST2PT leads to poor selection of the TROSY operators. 3F-Tyr spectra simulated with correct shuttling delays (a) and virtually cancelling delays for shuttling (1 ms) and high-field stabilization $(0 \mathrm{~ms})$ delays $(\mathbf{b})$. These simulations were performed for a global tumbling correlation time $\tau_{c}=25 \mathrm{~ns}$. Here, we show 15 contour levels starting from the maximum intensity and with a factor 1.2 between two consecutive levels. Peaks are labelled as Carbon-TROSY/-Fluorine TROSY $\left(\mathrm{C}^{\mathrm{T}} \mathrm{F}^{\mathrm{T}}\right)$, Carbon anti-TROSY/Fluorine-TROSY $\left(\mathrm{C}^{\mathrm{AT}} \mathrm{F}^{\mathrm{T}}\right)$, Carbon-TROSY/Fluorine anti-TROSY $\left(\mathrm{C}^{\mathrm{T}} \mathrm{F}^{\mathrm{AT}}\right)$ and Carbon anti-TROSY/Fluorine anti-TROSY $\left(\mathrm{C}^{\mathrm{AT}} \mathrm{F}^{\mathrm{AT}}\right)$. 
a)

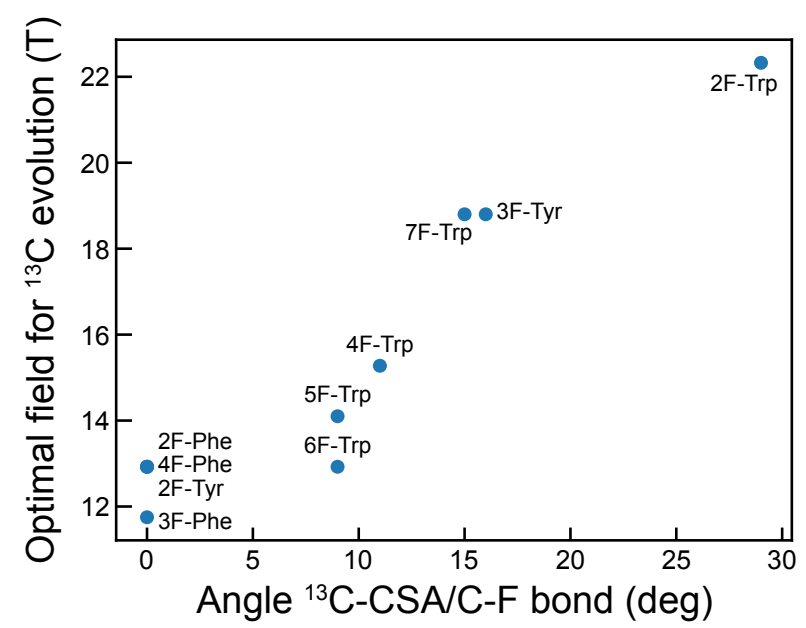

b)

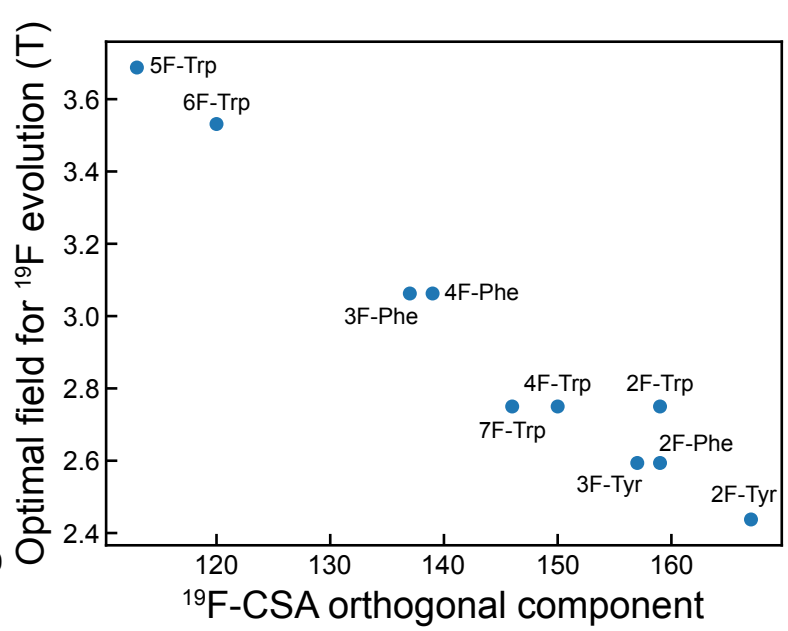

Figure S9: Correlation plot (a) between the angle between the ${ }^{13} \mathrm{C}-\mathrm{CSA}$ and the C-F bond and the optimal field for carbon-detection in the 2F-TROSY experiment, and (b) the orthogonal component of the ${ }^{19} \mathrm{~F}$-CSA $\left(\sigma_{\perp}^{F}\right.$, see TableS1) and the optimal field for fluorine chemical shift evolution in the $2 \mathrm{~F}$ TROSY experiment. Optimal fields are obtained for $\tau_{c}=100 \mathrm{~ns}$. Two examples are shown here for the determination of the optimal fields: the 3F-Tyr in the main text, and the 4F-Phe (Fig. S10).
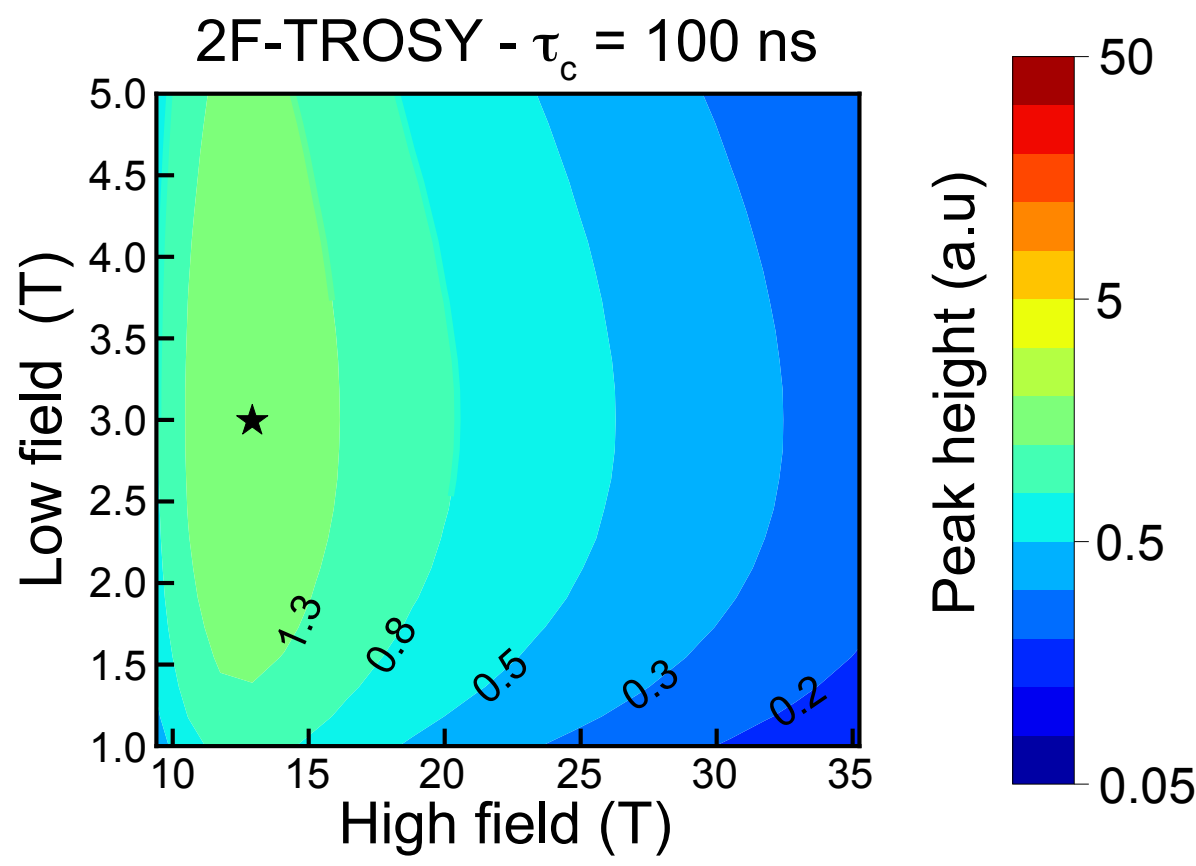

Figure S10: Magnetic field optimization of the 2F-TROSY experiment for the 4F-Phe. Expected peak height for a blobal tumbling correlation time $\tau_{c}=100 \mathrm{~ns}$. The color scale is identical as in the main text. The highest peak-height position is indicated with a star and reported in Fig. S9. 


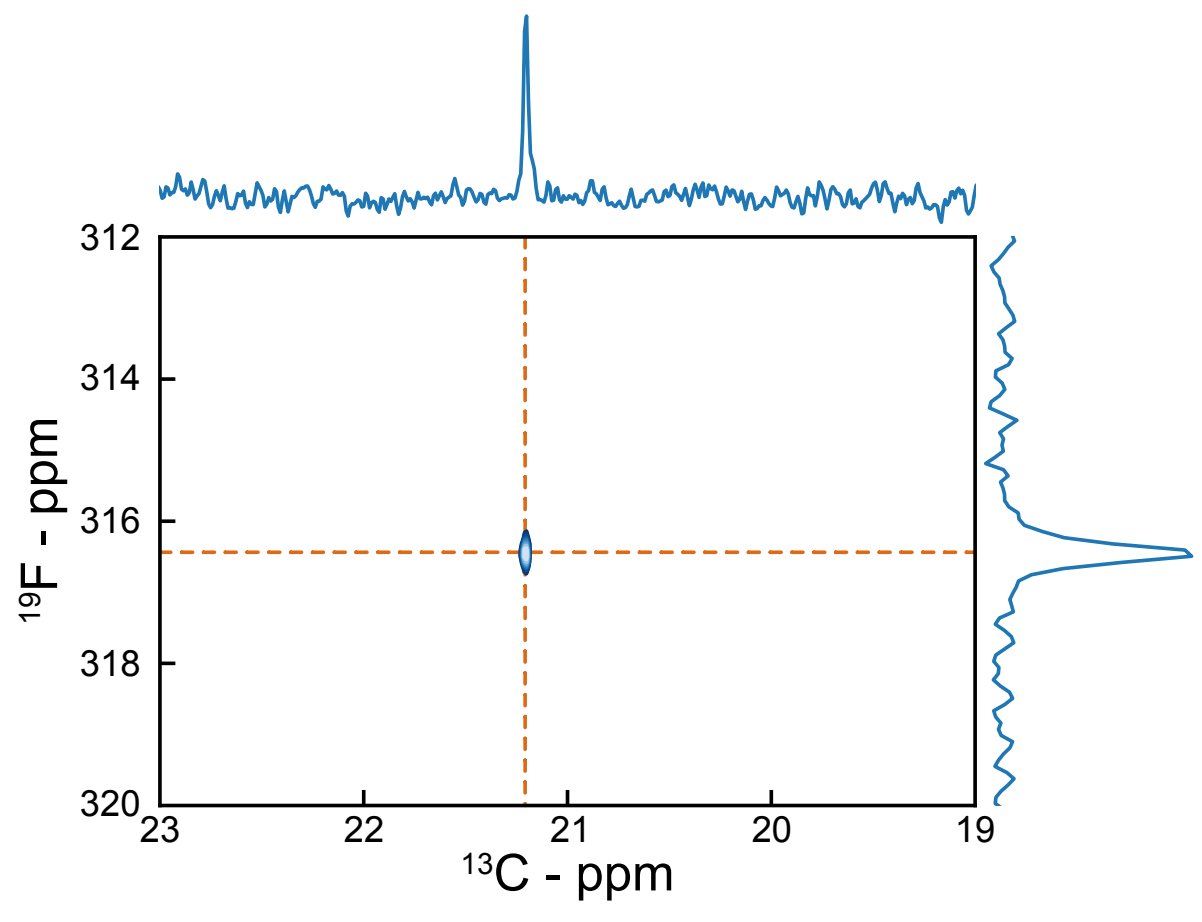

Figure S11: Simulation of the spectrum of MBP labeled on 3F-Tyr using the 1F-TROSY pulse sequence with experimental parameters identical to Ref. [7]: $B_{0}=14.1 \mathrm{~T}, 80$ scans, recycling delay of $2 \mathrm{sec}$, $\mathrm{t}_{1, \max }=10 \mathrm{~ms}, \mathrm{t}_{2, \max }=348 \mathrm{~ms}, 116(4,096)$ complex points in the indirect (direct) dimension. Noise was added in these simulations. We show 10 contour levels starting from the highest intensity and with a factor 1.2 between two consecutive levels. The signal to noise ratio is equal to 25.1. 
$t_{1, \max }$ multiplication factor

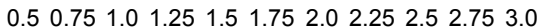

a)

1F-TROSY $-\tau_{c}=25 \mathrm{~ns}$

b)

$2 \mathrm{~F}-\mathrm{TROSY}-\tau_{\mathrm{c}}=25 \mathrm{~ns}$
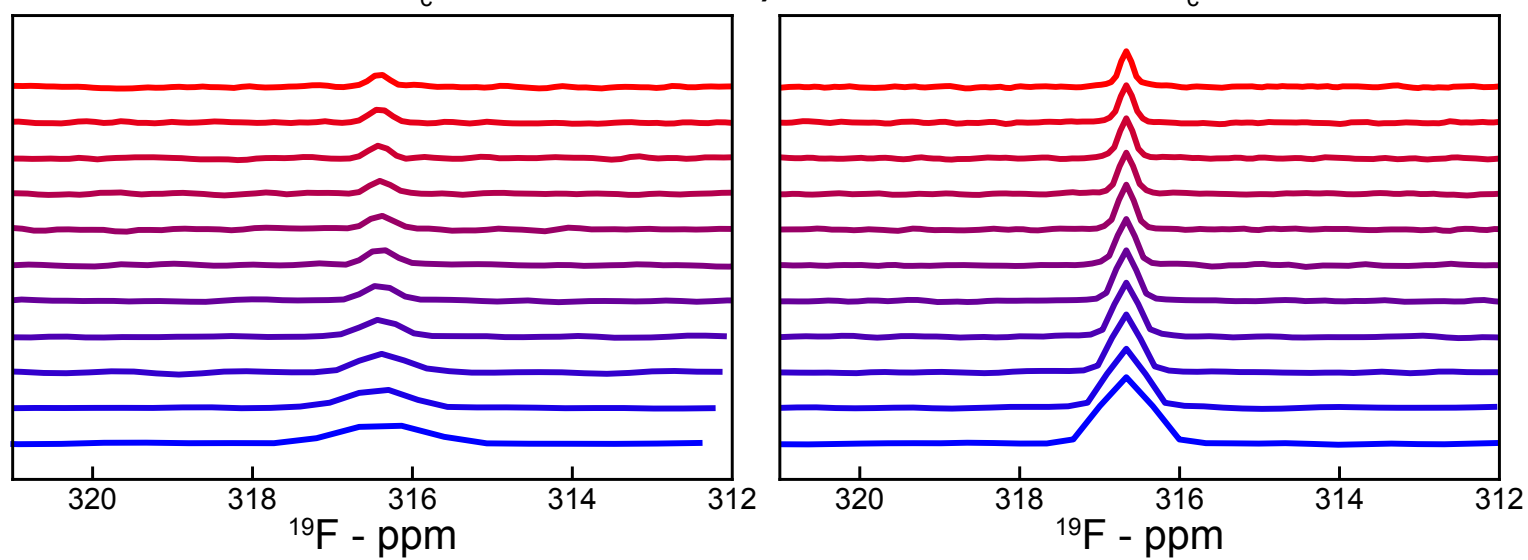

c)

1F-TROSY $-\tau_{c}=100 \mathrm{~ns}$

d)

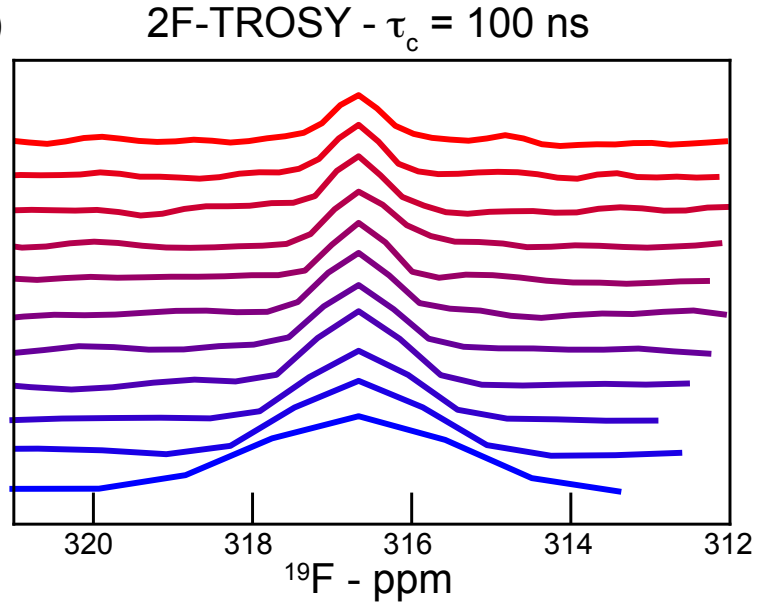

Figure S12: Simulated ${ }^{19} \mathrm{~F}$ cross sections in the presence of noise for the $3 \mathrm{~F}-\mathrm{Tyr} .{ }^{19} \mathrm{~F}$ cross sections for the 1F-TROSY (a, c) and 2F-TROSY (b, d) with a global tumbling correlation time $\tau_{c}=25 \mathrm{~ns}(\mathbf{a}, \mathbf{b})$ and $\tau_{c}=100 \mathrm{~ns}(\mathbf{c}, \mathbf{d})$ for different values of $t_{1, \max }$ calculated as $t_{1, \max }(C)=C \times T_{2}\left({ }^{19} F\right)$ where $\mathrm{C}$ is a multiplication factor, ranging from 0.5 to 3.0 , and $T_{2}\left({ }^{19} F\right)$ is the transverse relaxation time for either the fluorine TROSY operator (1F-TROSY) or fluorine coherence (2F-TROSY). Panels $\mathbf{a}$ and $\mathbf{b}$ on one side, and $\mathbf{c}$ and $\mathbf{d}$ on the other, have the same ordinate axis scale. 


\section{$t_{1, \text { max }}$ multiplication factor}

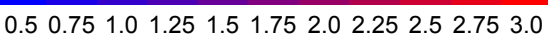
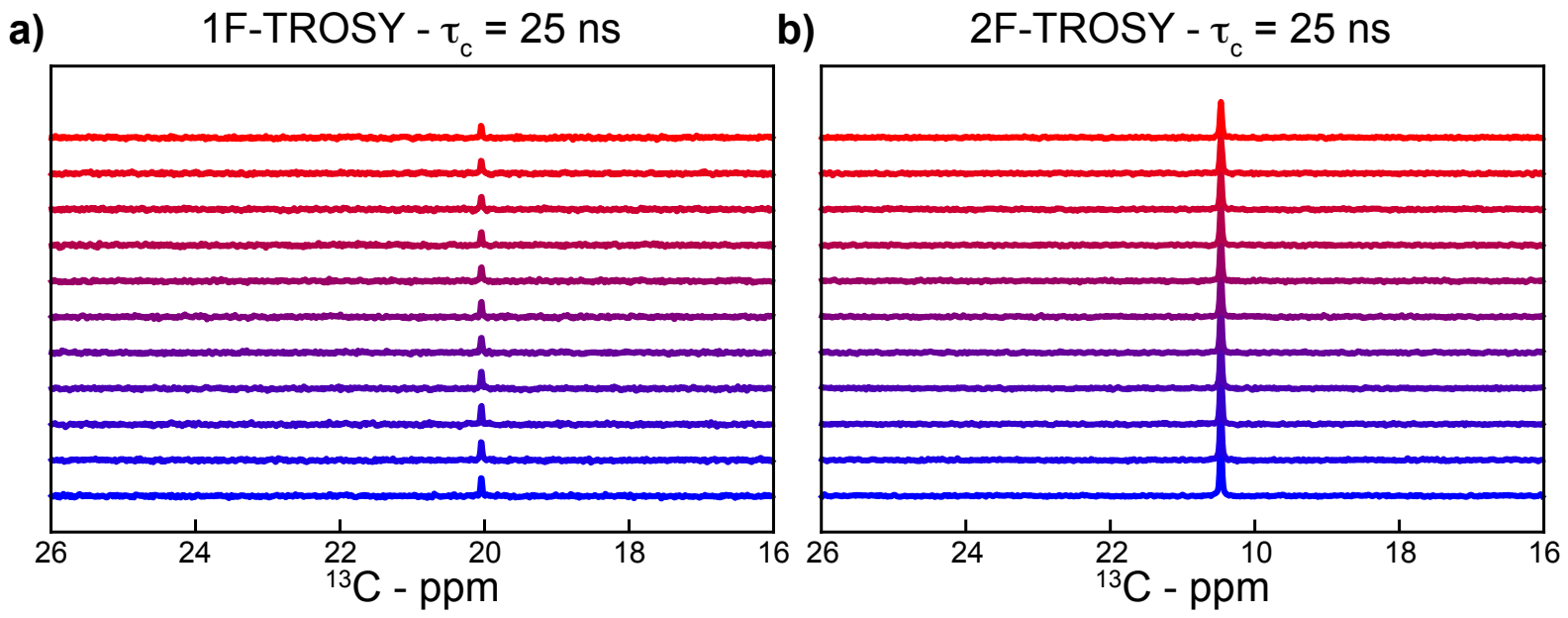

c)

1F-TROSY $-\tau_{c}=100 \mathrm{~ns}$

d)
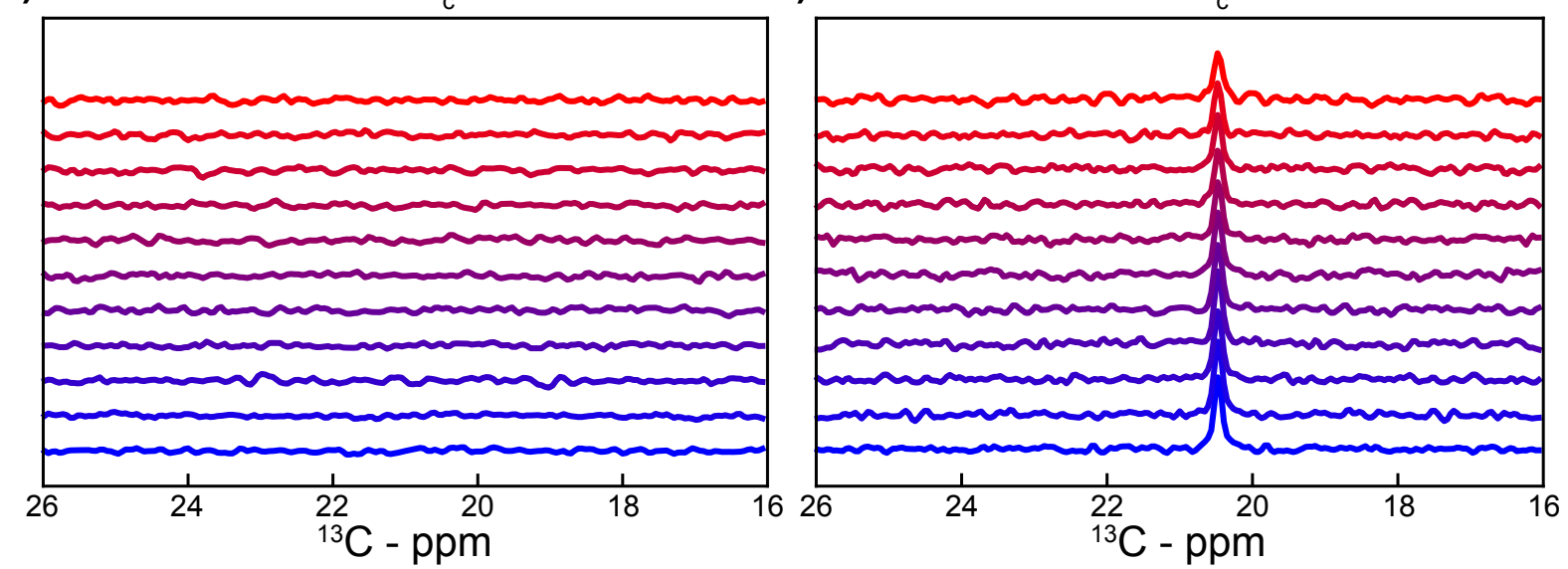

Figure S13: Simulated ${ }^{13} \mathrm{C}$ cross sections in the presence of noise for the $3 \mathrm{~F}-\mathrm{Tyr} .{ }^{13} \mathrm{C}$ cross sections for the 1F-TROSY $(\mathbf{a}, \mathbf{c})$ and 2F-TROSY $(\mathbf{b}, \mathbf{d})$ with a global tumbling correlation time $\tau_{c}=25 \mathrm{~ns}(\mathbf{a}, \mathbf{b})$ and $\tau_{c}=100 \mathrm{~ns}(\mathbf{c}, \mathbf{d})$ for different values of $t_{1, \max }$ calculated as $t_{1, \max }(C)=C \times T_{2}\left({ }^{19} \mathrm{~F}\right)$ where $\mathrm{C}$ is a multiplication factor, ranging from 0.5 to 3.0 , and $T_{2}\left({ }^{19} F\right)$ is the transverse relaxation time for either the fluorine TROSY operator (1F-TROSY) or fluorine coherence (2F-TROSY). Panels a and $\mathbf{b}$ on one side, and $\mathbf{c}$ and $\mathbf{d}$ on the other, have the same ordinate axis scale. 
a)

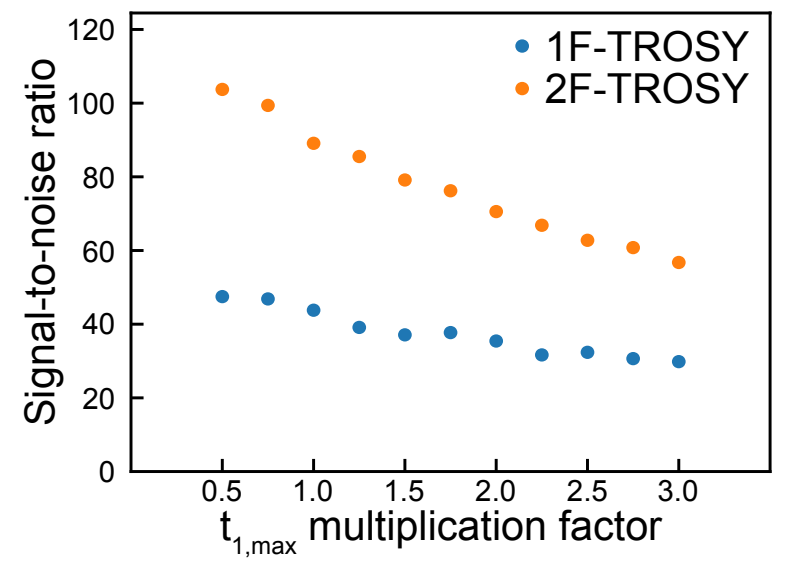

b) $\quad \tau_{\mathrm{c}}=100 \mathrm{~ns}$

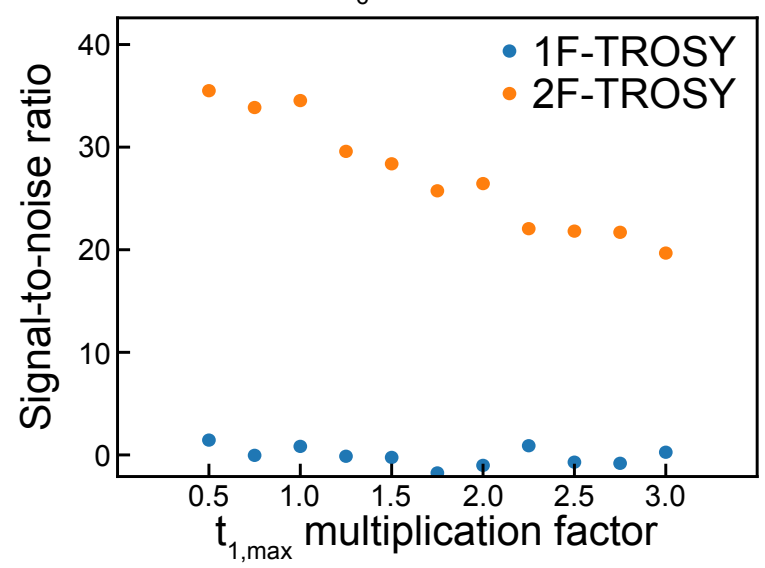

Figure S14: Signal-to-noise ratio for ${ }^{13} \mathrm{C}-{ }^{19} \mathrm{~F}$ TROSY spectra of $4 \mathrm{~F}-\mathrm{Phe}$. Signal-to-noise ratio (SNR) with a global tumbling correlation time $\tau_{c}=25 \mathrm{~ns}(\mathbf{a})$ and $\tau_{c}=100 \mathrm{~ns}(\mathbf{b})$. The experimental time is the same in each simulated experiment (4.9 hours). 


\section{References}

[1] N. Bolik-Coulon, P. Kadeřávek, P. Pelupessy, J.-N. Dumez, F. Ferrage, and S. F. Cousin J. Magn. Reson, vol. 313, p. 106718, 2020.

[2] A. Boeszoermenyi, S. Chhabra, A. Dubey, R. D. L., N. T. Burdzhiev, C. D. Chanev, O. I. Petrov, V. M. Gelev, M. Zhang, C. Anklin, H. Kovacs, G. Wagner, I. Kuprov, K. Takeuchi, and H. Arthanari Nat. Methods, vol. 16, p. 333, 2019.

[3] J. J. Helmius and C. P. Jaroniec J. Biomol. NMR, vol. 55, p. 355, 2013.

[4] K. V. Pervushin, G. Wider, and Wüthrich J. Biomol. NMR, vol. 12, p. 345, 1998.

[5] S. S. F. Cousin, P. Kadeřávek, B. Haddou, C. Charlier, T. Marquardsen, J.-M. J.-M. Tyburn, P.-A. P.-A. Bovier, F. Engelke, W. Maas, G. Bodenhausen, P. Pelupessy, and F. Ferrage Angew. Chem. Int. Ed., vol. 55, p. 9886, 2016.

[6] S. F. Cousin, C. Charlier, P. Kadeřávek, T. Marquardsen, J.-M. Tyburn, P.-A. Bovier, S. Ulzega, T. Speck, D. Wilhelm, F. Engelke, W. Maas, D. Sakellariou, G. Bodenhausen, P. Pelupessy, and F. Ferrage Phys. Chem. Chem. Phys., vol. 18, p. 33187, 2016.

[7] K. Takeuchi, H. Arthanari, and G. Wagner J. Biomol. NMR, vol. 66, p. 221, 2016. 